\title{
THE
}

6-8-2000

\section{Interference of Bulk and Boundary Scattering in Films with Quantum Size Effect}

\author{
A. E. Meyerovich \\ University of Rhode Island, sfo101@uri.edu
}

A. Stepaniants

University of Rhode Island

Follow this and additional works at: https://digitalcommons.uri.edu/phys_facpubs

Terms of Use

All rights reserved under copyright.

\section{Citation/Publisher Attribution}

Meyerovich, A. E., \& Stepaniants, A. (2000). Interference of Bulk and Boundary Scattering in Films with Quantum Size Effect. J. Phys.: Condens. Matter., 12, 5575-5597. doi: 10.1088/0953-8984/12/26/305 Available at: http://dx.doi.org/10.1088/0953-8984/12/26/305 


\title{
Interference of Bulk and Boundary Scattering in Films with Quantum Size Effect
}

\author{
A. E. Meyerovich, and A. Stepaniants \\ Department of Physics, University of Rhode Island, Kingston, RI 02881, USA \\ (June 8, 2000)
}

Typeset using REVTEX 


\begin{abstract}
The interference between boundary and bulk scattering processes is analyzed for ultrathin films with random rough walls. The effective collision and transport relaxation times for scattering by random bulk and surface inhomogeneities are calculated, when possible analytically, in quantum size effect conditions. The transport and localization results are expressed via the bulk transport parameters and statistical characteristics of the surface corrugation. The diagrammatic calculation includes second order effects for boundary scattering and full summation for bulk processes. The interference contribution is large in systems with robust bulk scattering and can be comparable, or even exceed, the pure wall contribution to the transport coefficients.
\end{abstract}

\title{
I. INTRODUCTION
}

Explosive developments in micro- and nanofabrication, multilayer systems, ultraclean materials, etc., resulted in proliferation of ballistic or semi-ballistic films. It is difficult to overestimate the role of boundary scattering for transport in such thin films (for early reviews see Refs. [1-3]).

Proper transport theory should not simply combine bulk and surface scattering, but has to include the interference between these scattering channels. Usually, this is not done. There are two obvious interference mechanisms: simultaneous presence of surface and bulk scattering centers within the same "reaction zone" and the correlation (or lack of thereof) between consecutive or multiple scattering processes. [Since the localization length in quasi$2 D$ systems is exponentially large, the paper deals mostly with interference contributions to "usual" diffusion. The localization-related phenomena are discussed in the end of the paper].

The interference should be large in quantum systems. In ultrathin films, the spatial confinement of motion perpendicular to the walls leads to the quantum size effect (QSE) 
- to the quantization of momentum and to the split of the $3 D$ spectrum $\epsilon(\mathbf{p})$ into a set of minibands $\epsilon_{j}(\mathbf{q})$ ( $\mathbf{q}$ is the momentum along the film). QSE is responsible for a sawlike dependence of the conductivity of metal films on the film thickness and/or the Fermi momentum irrespective of whether electrons are scattered in the bulk [4] or on the surface [5-10]. In non-degenerate semiconductor films with QSE this dependence is more smooth because of a more uniform occupancy of quantized states. Though we do not know of any direct observation of such saw- or step-like curves in transport measurements without magnetic field (with the exception of, maybe, Ref. [11]), QSE has been observed in both metal and semiconductor films (see, e.g., Refs. [12,13] and references therein) by scanning tunnel microscopy [14], ultraviolet electron spectroscopy, photoemission, and dozens of other optical experiments (Refs. $[3,15]$ and references therein). Recently QSE has also been observed in confined ${ }^{3} \mathrm{He}$ at ultralow temperatures [16]. The interference between bulk and surface scattering processes in quantized films has not been studied at all though the effect should be stronger than in quasiclassical films.

The interference between various bulk scattering processes in transport is well understood. The interference between the bulk and surface scattering is largely ignored, not because it is small, but simply because these processes are usually described by separate theoretical techniques. The bulk scattering is described by the collision operator which can include the interference between the bulk scattering channels but does not contain surface contributions at all. The surface scattering in transport is treated, instead, as a boundary condition. The (quasiclassical) transport equation operates with the distribution functions and does not contain particle phases explicitly: all phase-related information has already been processed during the derivation of the collision operator. Therefore, whenever the boundary scattering is introduced as a boundary condition for the transport equation, the boundary - bulk interference is already lost. Thus, the "standard" quasiclassical or quantum transport equation cannot account for the interference between the bulk and boundary processes and treats the bulk- and surface-driven relaxations, $\tau_{b}$ and $\tau_{w}$, as independent, 


$$
\tau_{e f f}^{-1}=\tau_{b}^{-1}+\tau_{w}^{-1}
$$

(the same for the mean free paths $\mathcal{L}_{e f f}, \mathcal{L}_{b}$, and $\mathcal{L}_{w}$ ). Note, that the bulk term $\tau_{b}^{-1}$ in this expression can contain the non-Matthiessen's interference between the bulk channels. To keep the interference between the bulk and boundary scattering, one should include the boundary scattering on the quantum mechanical level preceding the averaging which is responsible for the formation of the transport equation. Essentially, one should re-derive the transport equation starting from the scattering boundary conditions for the wave functions. This requires a simultaneous averaging over bulk and surface inhomogeneities.

The boundary scattering in thin films has been studied mostly for quasiclassical transport. The most common approaches, which are based on various modifications of the Fuchs-Sondheimer description, treat boundary and bulk scattering as independent. The experimental deviations from the Fuchs-Sondheimer picture are ascribed to the breakdown of the quasiclassical approach, usually without a conclusive identification of the particular breakdown mechanism. More sophisticated quasiclassical methods, which can reveal the interference, lead to almost intractable integral equations $[1,2]$.

The situation seems even worse in QSE conditions. Because of the complexity of the transport equation in quantized systems, the bulk-boundary interference has not been studied, so far, neither analytically nor numerically. The source of complexity is the large contribution of the off-diagonal terms. Only if the symmetry of the scattering vertex forbids the coupling of the longitudinal (diagonal) transport processes to off-diagonal terms, the quantum transport equation assumes the Waldmann-Snider form [17] which is, essentially, the result of the simplest quantization of the quasiclassical transport equation.

Recently, there have been several analytical [18-21] and numerical [22,23] attempts to describe transport in quantized films with both bulk and boundary scattering. Analytically, the bulk averaging and the averaging over surface inhomogeneities have been performed independently and the interference has been lost. The numerical simulations, which are, in general, not well suited for study of particle phases, also could not reveal the interference. 
This is very frustrating because the boundary-bulk interference is intuitively transparent and should be large in quantized systems.

Below we calculate the interference between surface and boundary scattering in transport in quantized films. The motivation is the large magnitude of the anticipated effect. The best way to reveal this interference is to derive the bulk and boundary collision operators simultaneously by the same technique. Bulk transport derivations are routinely done using the diagrammatic transport formalism [24,25]. Recently, we developed a similar formalism for quantized systems with scattering by random rough walls [26]. Since, by design, the derivation of the collision operator for surface scattering has been done in a bulk-like form, this formalism is well suited for the simultaneous study of bulk and boundary scattering.

The interference contribution to the conductivity is expressed below via the bulk transport parameters (the collision and transport relaxation times) and the statistical properties of surface roughness (the correlation function of surface inhomogeneities). The simplest way of presenting the results is in the form of a relative interference deviation from Eq.(1). As expected, the interference contribution in quantized systems is large and, sometimes, even exceeds the "pure" wall-driven term in (1).

Though the calculation is formally performed for a single-layer film with impenetrable rough walls, the results can be easily expanded to multilayer films with semi-transparent rough interlayer boundaries (see the end of Sec. II). The final results are illustrated for degenerate electron system such as metal films; the calculation for non-degenerate semiconductors is similar. The extension to other systems can be done in the same way as it has been done in Ref. [27] for quantized ballistic systems without bulk scattering.

In Section II we outline our approach and give the equation for the collision relaxation time $\tau_{\text {eff }}$ which includes the interference between the bulk and boundary scattering. The diagrammatic derivation of this equation is given in Appendix A. Appendix B contains auxiliary equations which can be useful for applications. In Section III we analyze, when possible analytically, the interference term in $\tau_{e f f}$ in different physical situations. In Section IV we calculate and analyze the transport time $\tau_{t r}$ using the equations of Appendix $\mathrm{C}$ for 
the diffusion propagator. The expressions for the transport time $\tau_{t r}$ are more cumbersome than those for the collision time $\tau_{e f f}$. Both the effective collision and transport times can be used for the evaluation of the localization length in quantized quasi-2D films (Sec. IV). Section $V$ contains a brief summary of the results.

\section{MAPPING TRANSFORMATION METHOD}

Simultaneous study of bulk and boundary scattering can be done by the mapping transformation method which maps the random boundary scattering problem onto an equivalent problem with ideal boundaries and randomly distorted bulk. This approach to ballistic transport was suggested first by Tesanovic et al [28] and, later, by Trivedi and Ashcroft [5] without an explicit expression for the mapping transformation. Independently, S. Stepaniants and one of the authors [29] and, later, Bratkovsky and Rashkeev [30] introduced the relevant Migdal-like transformation and performed explicit transport and localization calculations for ballistic systems (see also Refs. [26,27] and references therein; similar transformation was also used in Ref. [31] for calculation of the QSE spectrum in rough contacts).

This method should be modified for a system with bulk impurities $u\left(\mathbf{r}-\mathbf{r}_{i}\right)$,

$$
\widehat{H}_{0}=p^{2} / 2 m+U(\mathbf{r}), U(\mathbf{r})=\sum_{i} u\left(\mathbf{r}-\mathbf{r}_{i}\right)
$$

and two random rough walls,

$$
x_{1,2}= \pm L / 2 \mp \xi_{1,2}(y, z),\left\langle\xi_{1,2}\right\rangle=0,
$$

with the average clearance $L$. The volume average of the impurity field can be included into the chemical potential $\mu$ making $\left\langle u\left(\mathbf{r}-\mathbf{r}_{i}\right)\right\rangle_{V}=0$. The range of impurity potential is short in comparison to the average distance between impurities, $r_{0} \ll N_{i m p}^{-1 / 3}$, and impurities are not correlated with each other,

$$
\left\langle u\left(\mathbf{r}-\mathbf{r}_{i}\right) u\left(\mathbf{r}-\mathbf{r}_{j}\right)\right\rangle_{V}=\frac{1}{V} \delta_{i j} \int\left[u\left(\mathbf{r}-\mathbf{r}_{i}\right)\right]^{2} d \mathbf{r}_{i}
$$


In contrast to this, the correlation radius of the surface inhomogeneities $R$ which determines the decay of the correlation function,

$$
\begin{aligned}
\zeta_{i k}(|\mathbf{s}|) & =\left\langle\xi_{i}\left(\mathbf{s}_{1}\right) \xi_{k}\left(\mathbf{s}_{1}+\mathbf{s}\right)\right\rangle \equiv \int \xi_{i}\left(\mathbf{s}_{1}\right) \xi_{k}\left(\mathbf{s}_{1}+\mathbf{s}\right) d \mathbf{s}_{1} \\
\zeta_{i k}(\mathbf{q}) & =\int d \mathbf{s} e^{i \mathbf{q} \cdot \mathbf{s}} \zeta_{i k}(\mathbf{s})
\end{aligned}
$$

can be large ( $\mathbf{s}$ and $\mathbf{q}$ are the $2 D$ vectors in the plane of the wall $y, z$ ). Experimentally, this correlation function can have various shapes [32,33]. Analytically, we do not have to specify the form of this correlator. In numerical examples, we use the Gaussian correlator,

$$
\zeta_{i k}(\mathbf{s})=a_{i k} \ell^{2} \exp \left(-s^{2} / 2 R_{i k}^{2}\right), \zeta_{i k}(\mathbf{q})=2 \pi a_{i k} \ell^{2} R_{i k}^{2} \exp \left(-q^{2} R_{i k}^{2} / 2\right)
$$

where $\ell$ shows the scale of inhomogeneities, $a_{i k}$ are dimensionless amplitudes. To minimize the number of parameters, assume that the correlation radii for both walls are the same, $R_{i k}=R$, while the amplitudes $a_{i k}$ can remain different.

The standard requirement for calculations with the single-particle density matrix is $p_{0} \mathcal{L}_{\text {eff }} \gg 1\left(p_{0}\right.$ is the characteristic momentum of particles, $\mathcal{L}_{\text {eff }}$ is the mean free path; here and below $\hbar=1$ ). This is our only restriction on the particle wavelength. The surface roughness is assumed to be slight, $\ell \ll L, R(\ell$ and $R$ are the characteristic height and size of the surface inhomogeneities), and is described perturbatively. [Transport processes in systems with strong roughness, $\ell \sim L$ and/or $\ell \gtrsim R$, are trivial: a single collision with the wall dephases the particle completely]. There is no restriction on $p_{0} \ell$ or $p_{0} R$. The bulk scattering is not assumed to be weak. To get useful results for the boundary-bulk interference, one should start form a compact description of independent boundary- and bulk-dominated transport processes. For the boundary-dominated transport, we use the results [27] for ballistic particles in quantized films. For the bulk-dominated scattering in quantized films, a compact description exists only in the Born approximation or for a short-range interaction radius $r_{0} \ll L, R$ [elsewhere, the exact transport results contain the full bulk scattering vertex $\widehat{\Gamma}$ making them useless for applications]. Summarizing, we consider the films with

$$
p_{0} \mathcal{L}_{e f f} \gg 1, \quad \ell, r_{0} \ll L, R, \mathcal{L}_{b}
$$


These not very restrictive inequalities should be supplemented by the conditions of the quasiclassical motion along the walls and the absence of quantum resonances for quantized motion perpendicular to the walls. The former condition is standard. The latter one is discussed in detail in Ref. [26] according to which the resonance region is narrow.

Our diagrammatic technique [26] is based on the mapping transformation $\mathbf{r} \rightarrow \mathbf{R}$,

$$
X=\frac{x+\xi_{1} / 2-\xi_{2} / 2}{1-\xi_{1} / L-\xi_{2} / L}, Y=y, Z=z
$$

which makes both walls (3) flat, $X= \pm L / 2$.

The conjugate momentum transformation $\mathbf{p} \rightarrow \mathbf{P}$ identifies the effective random bulk distortion $\hat{V}\left\{\xi_{1,2}\right\}$ which, in the case of quadratic Hamiltonian (2), has the form [26,29]

$$
\begin{aligned}
\widehat{H} & =\frac{P^{2}}{2 m}+U(\mathbf{R})+\hat{V}+\delta U, \widehat{V}=\frac{\xi_{+}}{m L} \widehat{P}_{x}^{2}+\widehat{V}_{y}+\widehat{V}_{z}, \\
\widehat{V}_{y, z} & =\frac{1}{2 m}\left(\left(\frac{X}{L} \xi_{+y, z}^{\prime}-\frac{1}{2} \xi_{-y, z}^{\prime}\right) \widehat{P}_{x} \widehat{P}_{y, z}+\widehat{P}_{y, z}\left(\frac{X}{L} \xi_{+y, z}^{\prime}-\frac{1}{2} \xi_{-y, z}^{\prime}\right) \widehat{P}_{x}\right), \\
\delta U & =\sum\left[u\left(\mathbf{S}-\mathbf{s}_{i}, X-x_{i}+\xi_{-} / 2-\xi_{+} X / L\right)-u\left(\mathbf{S}-\mathbf{S}_{i}, X-X_{i}\right)\right], \xi_{ \pm}=\xi_{2} \pm \xi_{1} .
\end{aligned}
$$

The Hamiltonian (9) is non-Hermitian. The reason is that the transformation (8) with unchanged coordinates along the walls, $Y=y, Z=z$, changes volume and has the Jacobian not equal to 1 . In principle, this issue can be addressed by transforming coordinates $y, z$ as well. The detailed study in Ref. [26] showed that this more rigorous approach does not have any noticeable effect on the results outside the quantum resonance domain.

The problem with the corrugated walls is now mapped onto the equivalent bulk problem with flat walls, $X=\mp L / 2$, and random bulk distortion $U+\hat{V}+\delta U(9)$. Unfortunately, this bulk distortion contains not only the term $\widehat{V}+\delta U$, which is proportional to the small wall roughness $\xi$, but also the purely bulk term $U$ which can be large. Without this large term, one could use the transport equation of Refs. [26,27] for QSE systems in the second order in the surface distortion $\xi$. Here, one needs a different equation, which is still of the second order in $\xi$ but contains the full summation of the impurity terms with $U$.

The results get cumbersome because of QSE which splits the $3 D$ spectrum $\epsilon(\mathbf{p})=p^{2} / 2 m$ into a set of $2 D$ minibands, $\epsilon_{j}(\mathbf{q})=(1 / 2 m)\left[(\pi j / L)^{2}+q^{2}\right]$ and leads to a matrix structure 
of equations. The diagrammatic series with the averaging over the surface inhomogeneities, $\langle\ldots\rangle_{\xi}$, and distribution of impurities, $\langle\ldots\rangle_{V}$, is analyzed in Appendix A. In the end, the effective relaxation time (the imaginary part of the self energy, $\Sigma^{A}-\Sigma^{R}$ ) in the second order in $\xi$ and with full impurity summation reduces to (47),

$$
\frac{1}{\tau_{j}^{(e f f)}(\mathbf{q})}=\frac{1}{\tau_{j}^{(b)}(\mathbf{q})}+\sum_{j^{\prime}=1}^{S} \int \frac{W_{j j^{\prime}}\left(\mathbf{q}, \mathbf{q}^{\prime}\right) / \tau_{j^{\prime}}^{(b)}\left(\mathbf{q}^{\prime}\right)}{\left(\epsilon_{j^{\prime}}\left(\mathbf{q}^{\prime}\right)-\mu\right)^{2}+\left(1 / 2 \tau_{j^{\prime}}^{(b)}\left(\mathbf{q}^{\prime}\right)\right)^{2}} \frac{d \mathbf{q}^{\prime}}{(2 \pi)^{2}}
$$

where $S$ is the number of occupied or energetically accessible minibands $\epsilon_{j}(\mathbf{q})$, and $\mu$, depending on the system, is the Fermi energy $E_{F}$ for degenerate fermions, particle energy $E$ for single particle systems, temperature for Boltzmann quantum gases, etc. The wall-induced transition probability $W_{j j^{\prime}}\left(\mathbf{q}, \mathbf{q}^{\prime}\right)$ is determined by the averaged square of the matrix element $\left\langle\left|V_{j j^{\prime}}\right|^{2}\right\rangle_{\xi}$ of the perturbation $\widehat{V}(9)$ and is equal to $[26]$

$$
W_{j j^{\prime}}\left(\mathbf{q}, \mathbf{q}^{\prime}\right)=\frac{\pi^{4}}{m^{2} L^{6}}\left(\zeta_{11}\left(\mathbf{q}-\mathbf{q}^{\prime}\right)+\zeta_{22}\left(\mathbf{q}-\mathbf{q}^{\prime}\right)+2(-1)^{j+j^{\prime}} \zeta_{12}\left(\mathbf{q}-\mathbf{q}^{\prime}\right)\right) j^{2} j^{\prime 2}
$$

QSE is responsible for the replacement of the bulk relaxation time $\tau_{b}(\mathbf{p})$ by the times $\tau_{j}^{(b)}(\mathbf{q})$ for individual minibands $\epsilon_{j}$. The exact values of $\tau_{j}^{(b)}(\mathbf{q})$ for thin films are unknown even if one knows the exact $3 D$ dependence $\tau_{b}(\mathbf{p})$. The expressions for $\tau_{j}^{(b)}(\mathbf{q})$ in the Born approximation are given in Appendix B.

The first term in Eq.(10) is the purely bulk term. The second one represents the contribution from collisions with the walls renormalized by bulk scattering processes. This renormalization is the sought-for interference contribution to the effective relaxation time. It is clear from of the diagrams for the self energy in Appendix A that the effective relaxation time has the form (10) irrespective of whether the bulk relaxation time $\tau_{j}^{(b)}(\mathbf{q})$ is associated with impurity or particle-particle scattering. [The key is that the first diagram in Figure 9 dominates over the second; this is always the case when the interaction potential is sufficiently short-range].

Formally, the above equations were obtained for ultrathin single-layer films with impenetrable external walls. In Ref. [27] we found how to describe, within the same method, 
the scattering by inhomogeneities of rough interlayer boundaries in ballistic multilayer systems. In the multilayer case without bulk scattering, the overall surface-related scattering probability $W_{j j^{\prime}}\left(\mathbf{q}, \mathbf{q}^{\prime}\right)$ is a sum of contributions from each individual wall plus interwall interference terms which disappear if there is no correlation between inhomogeneities from different walls. Each such term is given by the correlation function of inhomogeneities on the corresponding wall with a simple coefficient that reflects the permeability of the interlayer boundary and the overall structure of the system. Straightforward analysis shows that if the bulk scattering within all layers is the same, then the effective relaxation time $\tau_{j}^{(e f f)}(\mathbf{q})$ is still determined by Eq.(10) with $W_{j j^{\prime}}\left(\mathbf{q}, \mathbf{q}^{\prime}\right)$ given not by Eq.(11), but by a similar equation of Ref. [27] for multilayer systems. If the bulk scattering in different layers is not the same, then the calculations should be performed for each layer separately. The final results for multilayer systems are qualitatively similar to those for single-layer films but involve additional parameters such as permeabilities and positions of interlayer boundaries, correlation radius and amplitude of inhomogeneities on each boundary, etc. To avoid parameter clutter and have the results in the most transparent form, we show the results only for a single-layer systems with two rough walls.

\section{RELAXATION TIME}

\section{A. Effective relaxation time}

The "pure" wall relaxation time in ballistic systems [27],

$$
\frac{1}{\tau_{j}^{(w)}}=\sum_{j^{\prime}=1}^{S} \int \frac{d \mathbf{q}^{\prime}}{2 \pi} W_{j j^{\prime}}\left(\mathbf{q}, \mathbf{q}^{\prime}\right) \delta\left(\epsilon_{j^{\prime}}\left(\mathbf{q}^{\prime}\right)-\mu\right),
$$

corresponds to Eq.(10) with $\tau_{j}^{(b)} \rightarrow \infty$. Thus, the interference contribution $\tau^{(i n t)}$ to the effective relaxation time $\tau^{(e f f)}$ is determined by the difference of Eqs. (1) and (10):

$$
\frac{1}{\tau_{j}^{(i n t)}} \equiv \frac{1}{\tau_{j}^{(e f f)}}-\frac{1}{\tau_{j}^{(b)}}-\frac{1}{\tau_{j}^{(w)}}=\sum_{j^{\prime}=1}^{S} \int \frac{d \mathbf{q}^{\prime}}{(2 \pi)^{2}} W_{j j^{\prime}}\left(\mathbf{q}, \mathbf{q}^{\prime}\right)
$$




$$
\times\left[\frac{1 / \tau_{j^{\prime}}^{(b)}\left(\mathbf{q}^{\prime}\right)}{\left(\epsilon_{j^{\prime}}\left(\mathbf{q}^{\prime}\right)-\mu\right)^{2}+\left(1 / 2 \tau_{j^{\prime}}^{(b)}\left(\mathbf{q}^{\prime}\right)\right)^{2}}-2 \pi \delta\left(\epsilon_{j^{\prime}}\left(\mathbf{q}^{\prime}\right)-\mu\right)\right] .
$$

The integral in Eq.(13) depends on the relation between three length scales, $R, \mathcal{L}_{b}, L$. The numerator, $W_{j j^{\prime}}^{(0)}\left(q_{j}, q^{\prime}\right)(11)$, represents a peak in the momentum space $q^{\prime}$ which is centered around $q_{j}=\left(2 m \mu-(\pi j / L)^{2}\right)^{1 / 2}$ and has the width $1 / R$. For example, if the surface inhomogeneities are Gaussian (6), the zeroth angular harmonic of the scattering probabilities (11) over the angle between the vectors $\mathbf{q}$ and $\mathbf{q}^{\prime}$ is equal to

$$
\begin{aligned}
W_{j j^{\prime}}^{(0)}\left(q_{j}, q^{\prime}\right)= & \frac{2 \pi^{5} \ell^{2} R^{2}}{m^{2} L^{6}}\left(a_{11}+a_{22}+2 a_{12}(-1)^{j+j^{\prime}}\right) j^{2} j^{\prime 2} \exp \left[-\left(q_{j}-q^{\prime}\right)^{2} R^{2} / 2\right] \times \\
& {\left[{ }_{1} F_{1}\left(\frac{1}{2}, 2,-2 q_{j} q^{\prime} R^{2}\right)+{ }_{1} F_{1}\left(\frac{3}{2}, 2,-2 q_{j} q^{\prime} R^{2}\right)\right] }
\end{aligned}
$$

$\left({ }_{1} F_{1}\right.$ is the hypergeometric function). The denominator in the same integrand gives rise to another peak of the width $1 / \mathcal{L}_{b}$ centered around $q_{j^{\prime}}$. The separation of these two peaks, i.e., the distance between the points $q_{j}$ and $q_{j^{\prime}}$, is of the order of $\left|j-j^{\prime}\right| / L$ and involves the third spacial scale, $L$.

If there is a pronounced hierarchy of these three scales, the integral (15) can be calculated analytically. Otherwise, the effective relaxation time $\tau_{j}^{(e f f)}$ can be calculated numerically for any type of the surface correlation function $\zeta\left(\mathbf{q}, \mathbf{q}^{\prime}\right)$ provided that the bulk relaxation times $\tau_{j}^{(b)}$ are known.

The bulk scattering time $\tau_{j}^{(b)}(\mathbf{q})$ is defined by the impurity potential $u(\mathbf{r})$ with a short range $r_{0}$. This time changes with the change in $\mathbf{q}$ on the scale $1 / r_{0}$ which is slow, Eq.(7), in comparison with the rates $1 / R$ for the correlation decay of $W_{j j^{\prime}}\left(\mathbf{q}, \mathbf{q}^{\prime}\right)$ and $1 / \mathcal{L}_{b}$ for the collision decay of the denominator in Eq.(13). This means that $\tau_{j}^{(b)}(\mathbf{q})$ in Eq.(13) can be considered constant. What is more, for systems with a relatively narrow energy distribution such as degenerate fermions $p_{F} \mathcal{L} \gg 1$, single-particle systems $p_{0} \mathcal{L} \gg 1$, Boltzmann systems with $p_{T} \mathcal{L} \gg 1$, etc., we need the values of $\tau_{j}^{(e f f)}$ only at $q=q_{j}$. Then Eq.(13) can be rewritten as

$$
\frac{1}{\tau_{j}^{(i n t)}}=\frac{1}{4 \pi} \sum_{j^{\prime}=1}^{S} \frac{1}{\tau_{j^{\prime}}^{(b)}} \int \frac{W_{j j^{\prime}}^{(0)}\left(q_{j}, q^{\prime}\right) q^{\prime} d q^{\prime}}{\left(\epsilon_{j^{\prime}}\left(\mathbf{q}^{\prime}\right)-\mu\right)^{2}+\left(1 / 2 \tau_{j^{\prime}}^{(b)}\right)^{2}}-\frac{m}{2} \sum_{j^{\prime}=1}^{S} W_{j j^{\prime}}^{(0)}\left(q_{j}, q_{j^{\prime}}\right),
$$


where $W_{j j^{\prime}}^{(0)}$ is the zeroth angular harmonic of the transition probability $W_{j j^{\prime}}\left(q, q^{\prime}, \cos \theta\right)$ (11).

The dependence of the bulk relaxation $\tau_{j}^{(b)}$ on the miniband index $j$ is not always available. Even in the Born approximation (Appendix B) the exact dependence is known only for large indices $j \gg 1$ or for the short-rang interaction, $r_{0} \ll 1 / p_{0}, L, R$, Eq.(7). In these two cases, all $\tau_{j}^{(b)}$ are the same, $\tau_{j}^{(b)} \sim \tau_{b}$. Since the issue of the dependence of the bulk relaxation time $\tau_{j}^{(b)}$ on the band index $j$ in quantized systems in the context of this paper is peripheral anyway, we assume in all numerical calculations for $\tau^{(e f f)}$ that $\tau_{j}^{(b)}$ does not depend on $j$, $\tau_{j}^{(b)}=\tau_{b}$.

The relative interference contribution (15),(??) can be described by the dimensionless parameter $\chi$,

$$
\chi_{j} \equiv\left[\frac{1}{\tau_{j}^{(e f f)}}-\frac{1}{\tau_{b}}\right] \tau_{j}^{(w)}=1+\frac{\tau_{j}^{(w)}}{\tau_{j}^{(i n t)}},
$$

which is the ratio of two terms in r.h.s. of Eq.(??). The Matthiessen's rule (1), i.e., the lack of interference $\tau^{(i n t)} \rightarrow \infty$, corresponds to $\chi_{j}=1$. In the case of Gaussian correlation of surface corrugation (6), this relative contribution is

$$
\chi_{j}=\frac{\sum_{j^{\prime}} j^{\prime 2}\left[1+2(-1)^{j+j^{\prime}} a\right] \int d \epsilon F_{j}(q) \Delta_{j^{\prime}}(q)}{\sum_{j^{\prime}} j^{\prime 2}\left[1+2(-1)^{j+j^{\prime}} a\right] F_{j}\left(q_{j^{\prime}}\right)},
$$

where

$$
\begin{aligned}
F_{j}(q) & =\left[{ }_{1} F_{1}\left(\frac{1}{2}, 2,-2 q_{j} q R^{2}\right)+{ }_{1} F_{1}\left(\frac{3}{2}, 2,-2 q_{j} q R^{2}\right)\right] \exp \left[-R^{2}\left(q_{j}-q\right)^{2} / 2\right], \\
\Delta_{j^{\prime}}(q) & =\frac{1}{2 \pi} \frac{1 / \tau_{b}}{\left(\epsilon_{j^{\prime}}(q)-\mu\right)^{2}+\left(1 / 2 \tau_{b}\right)^{2}}, a=\frac{a_{12}}{a_{11}+a_{22}} .
\end{aligned}
$$

When $\tau_{b} \rightarrow \infty$, the function $\Delta_{j}(q) \rightarrow \delta\left(\epsilon_{j}(q)-\mu\right)$ and $\chi \rightarrow 1$. Figures $1-3$ demonstrate that the interference often, but not always, lowers the effective relaxation rate $\left(\chi_{j}<1\right)$, i.e., decreases the boundary contribution to transport coefficients. 


\section{B. Interference contribution: numerical results}

Numerical examples of the relative interference contribution $\chi_{j}$ to the effective relaxation time are given in Figures $1-3$. Figure 1 shows $\chi_{j}$ for three minibands $(j=1$ - thin line; $j=5$ - bold line; $j=9$ - dotted line) as a function of $p_{0} R$. The thickness of the film in units of $1 / p_{0}$ is $p_{0} L=30$, and the bulk mean free path $p_{0} \mathcal{L}_{b}=100$. This value of $p_{0} L$ corresponds to 9 energetically accessible minibands. When the bulk mean free path $\mathcal{L}_{b} \gg R, L$, the situation is close to Matthiessen's $(\chi \sim 1)$, but with increasing $R$ the interference effects become well-pronounced even though the mean free path is larger than the clearance between the walls, $\mathcal{L}_{b} / L=10$. 


\section{FIGURES}

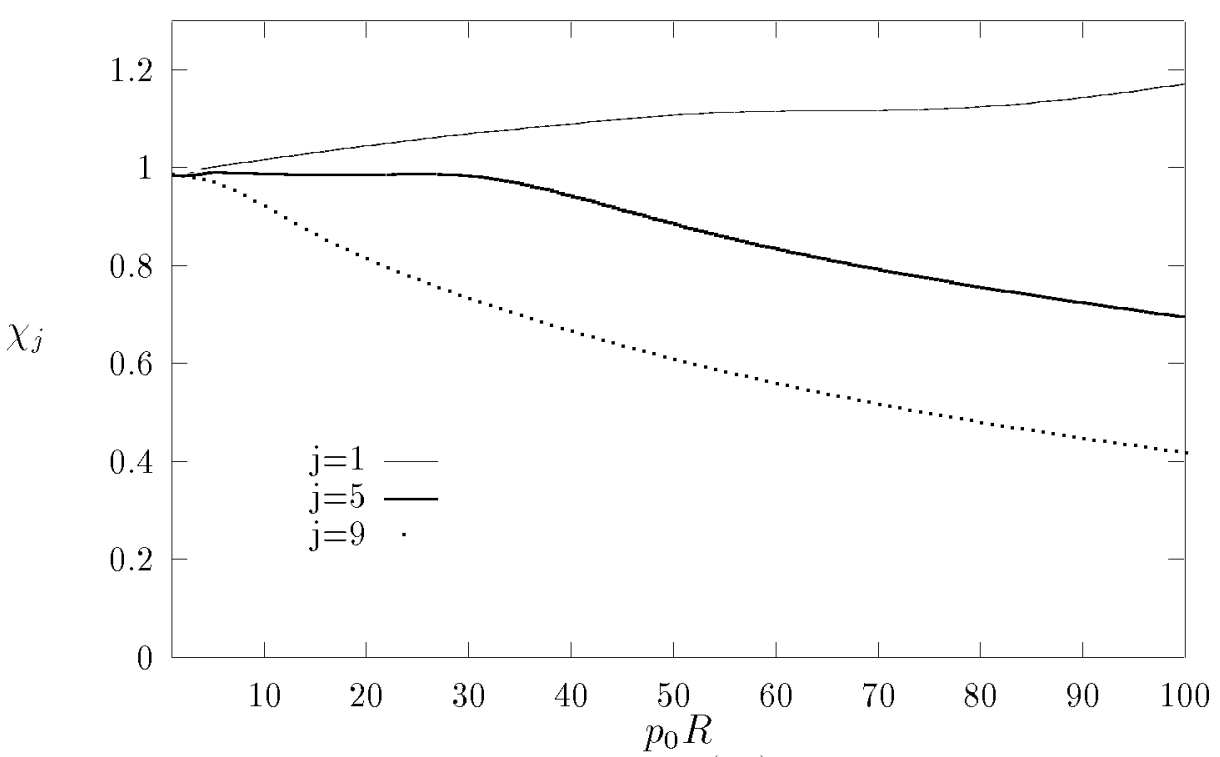

FIG. 1. Relative interference contribution $\chi_{j}$, Eq. (16), for $j=1 ; 5 ; 9$ as a function of the size of the surface inhomogeneities $p_{0} R$ for $p_{0} L=30$ and $p_{0} \mathcal{L}_{b}=100$

Figure 2 shows the dependence of $\chi_{j}(j=1$ - thin line; $j=16$ - bold line; $j=31$ dotted line) on $p_{0} \mathcal{L}_{b}$ at $p_{0} R=50$ and $p_{0} L=100$ (31 energetically accessible minibands). It is clear that when $\mathcal{L}_{b}$ becomes much larger than $L$ and $R$, the interference corrections disappear and $\chi_{j} \rightarrow 1$.

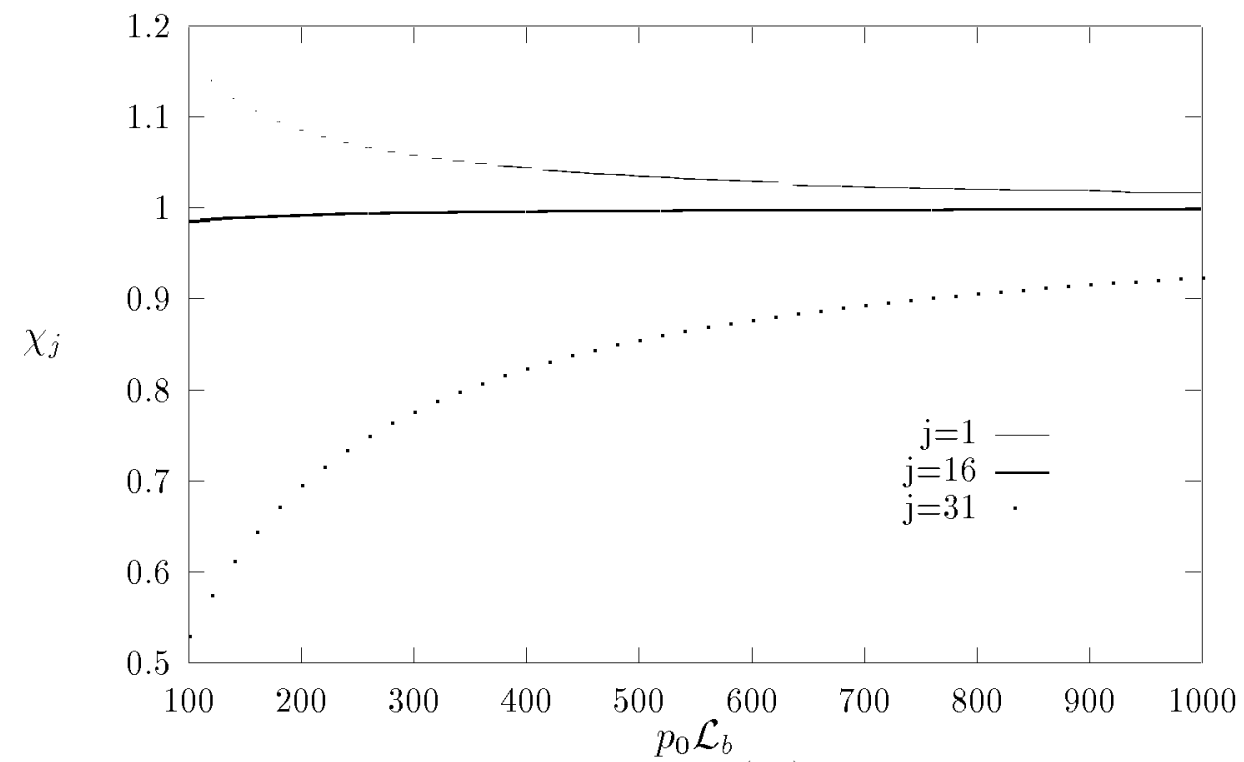

FIG. 2. Relative interference contribution $\chi_{j}$, Eq. (16), for $j=1 ; 16 ; 31$ as a function of the bulk free path $p_{0} \mathcal{L}_{b}$ for $p_{0} L=100$ and $p_{0} R=50$ 
Figure 3 shows the dependence of $\chi_{j}(j=1$ - thin line; $j=2$ - bold line; $j=3$ - dotted line) on $p_{0} L$ at $p_{0} R=5$ and $p_{0} \mathcal{L}_{b}=20$. The steps on the curves correspond to the changes in number of accessible minibands with increasing $p_{0} L$. As in ballistic systems [26,27], these singularities are pronounced at smaller $p_{0} R$, while their amplitude decreases with increasing film thickness $p_{0} L$.

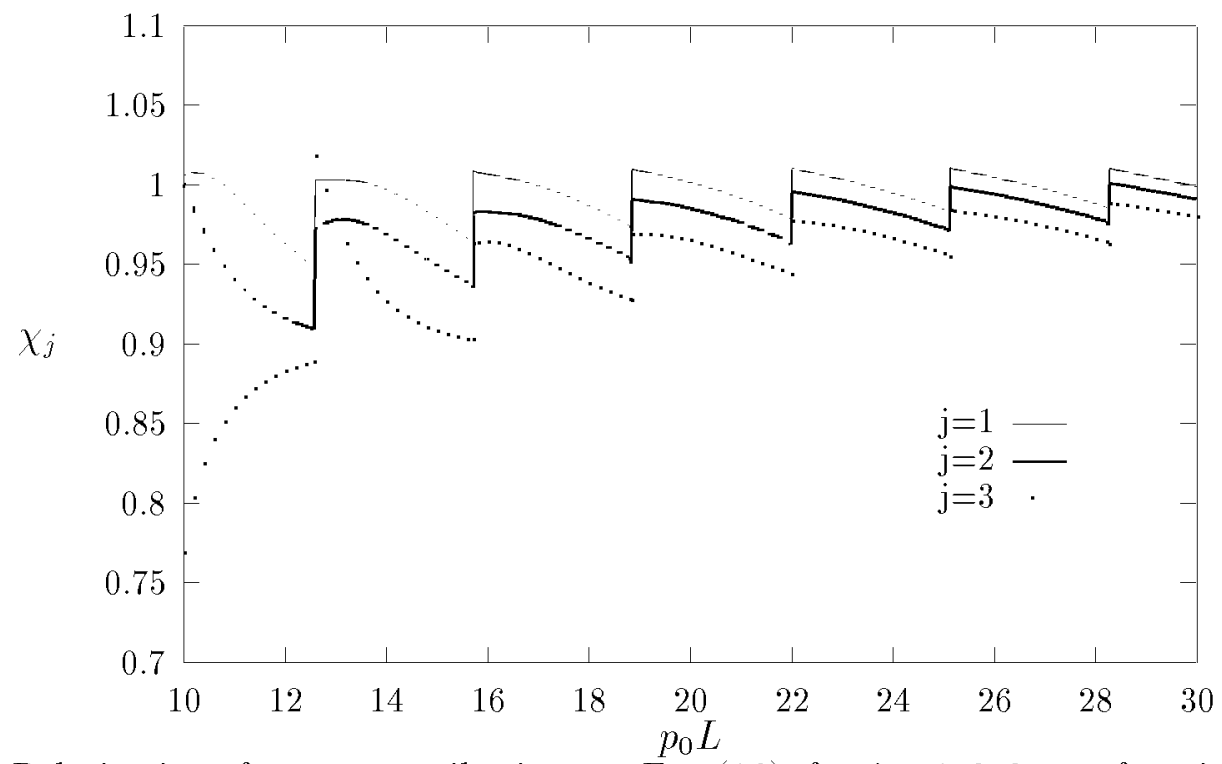

FIG. 3. Relative interference contribution $\chi_{j}$, Eq. (16), for $j=1 ; 2 ; 3$ as a function of the film thickness $p_{0} L$ for $p_{0} R=5$ and $p_{0} \mathcal{L}_{b}=20$

Under the certain conditions, the interference contribution to the relaxation time can be calculated analytically. These results are discussed in the next three subsections.

\section{Interference for large bulk mean free path}

If $\mathcal{L}_{b} \gg R$, the integrand in Eq.(10) behaves effectively as a $\delta$-function,

$$
\frac{1 / \tau_{j^{\prime}}^{(b)}}{\left(\epsilon_{j^{\prime}}\left(\mathbf{q}^{\prime}\right)-\mu\right)^{2}+\left(1 / 2 \tau_{j^{\prime}}^{(b)}\right)^{2}} \rightarrow 2 \pi \delta\left(\epsilon_{j^{\prime}}\left(\mathbf{q}^{\prime}\right)-\mu\right),
$$

and the deviation (15) from the Matthiessen's rule disappears while the wall-induced relaxation time is equal to

$$
\frac{1}{\tau_{j}^{(w)}}=\frac{\pi^{4}}{2 m L^{6}} \sum_{j^{\prime}} j^{2} j^{\prime 2}\left(\zeta_{11}^{(0)}\left(q_{j}, q_{j^{\prime}}\right)+\zeta_{22}^{(0)}\left(q_{j}, q_{j^{\prime}}\right)+2(-1)^{j+j^{\prime}} \zeta_{12}^{(0)}\left(q_{j}, q_{j^{\prime}}\right)\right) \text {. }
$$


In this limit, the bulk and wall scattering processes are truly independent.

The summation in Eq.(20) can be performed analytically in two limiting cases. When the film thickness is much smaller than the correlation length, $R \gg L$, the gaps between the minibands are so large that the scattering by the surface inhomogeneities cannot cause interband transitions and the diagonal elements of the matrix $W_{j j^{\prime}}$ are much larger than the off-diagonal ones, $W_{j j^{\prime}} \simeq \delta_{j j^{\prime}} W_{j j}$. In this case,

$$
\frac{1}{\tau_{j}^{(w)}}=\frac{\pi^{4}}{2 m L^{6}} j^{4}\left(\zeta_{11}^{(0)}\left(q_{j}, q_{j}\right)+\zeta_{22}^{(0)}\left(q_{j}, q_{j}\right)+2 \zeta_{12}^{(0)}\left(q_{j}, q_{j}\right)\right)
$$

or, for Gaussian correlations,

$$
\frac{1}{\tau_{j}^{(w)}}=\frac{\pi^{5} \ell^{2} R^{2}}{m L^{6}} j^{4}\left(a_{11}+a_{22}+2 a_{12}\right)\left[{ }_{1} F_{1}\left(\frac{1}{2}, 2,-2 q_{j}^{2} R^{2}\right)+{ }_{1} F_{1}\left(\frac{3}{2}, 2,-2 q_{j}^{2} R^{2}\right)\right] .
$$

For long-wave particles, $p_{0} R \ll 1$, the scattering cross-section for surface inhomogeneities of the size $R$ is a constant independent of momenta (quantum reflection), $W\left(\mathbf{q}-\mathbf{q}^{\prime}\right) \simeq$ $W(0)$, and Eq.(20) becomes

$$
\frac{1}{\tau_{j}^{(w)}}=\frac{\pi^{4}}{6 m L^{6}} j^{2} S(S+1)(2 S+1)\left(\zeta_{11}^{(0)}(0)+\zeta_{22}^{(0)}(0)+\frac{6(-1)^{j+S}}{2 S+1} \zeta_{12}^{(0)}(0)\right),
$$

where $S$ is the number of occupied or energetically accessible minibands. The Gaussian equivalent is

$$
\frac{1}{\tau_{j}^{(w)}}=\frac{2 \pi^{5} \ell^{2} R^{2}}{3 m L^{6}} j^{2} S(S+1)(2 S+1)\left(a_{11}+a_{22}+\frac{6(-1)^{j+S}}{2 S+1} a_{12}\right) .
$$

\section{Interference for small bulk mean free path}

In all other situations, the interference between boundary and bulk scattering is large. When the bulk mean free path is small, $\mathcal{L}_{b} \ll R$, the numerator in the integrand (15) is a peak which is narrow on the scale of the change in denominator, and the denominator can be pulled out of the integral,

$$
\frac{1}{\tau_{j}^{(e f f)}}-\frac{1}{\tau_{j}^{(b)}}=\sum_{j^{\prime}} \frac{1 / \tau_{j^{\prime}}^{(b)}}{\left(\epsilon_{j^{\prime}}\left(q_{j}\right)-\mu\right)^{2}+\left(1 / 2 \tau_{j^{\prime}}^{(b)}\right)^{2}} \int W_{j j^{\prime}}^{(0)}\left(q_{j}, q^{\prime}\right) \frac{q^{\prime} d q^{\prime}}{4 \pi} .
$$


This integral is nothing but the Fourier image of $W_{j j^{\prime}}\left(q_{j}, q^{\prime}\right), W_{j j^{\prime}}(\mathbf{s})$ at $s=0$ :

$$
\frac{1}{\tau_{j}^{(e f f)}}-\frac{1}{\tau_{j}^{(b)}}=\frac{4 \pi^{4}}{L^{2}} \sum_{j^{\prime}} \frac{\left(\zeta_{11}(\mathbf{s}=0)+\zeta_{22}(\mathbf{s}=0)+2(-1)^{j+j^{\prime}} \zeta_{12}(\mathbf{s}=0)\right) j^{2} j^{\prime 2} \tau_{j^{\prime}}^{(b)}}{\pi^{4}\left(j^{2}-j^{\prime 2}\right)^{2}\left(\tau_{j^{\prime}}^{(b)}\right)^{2}+m^{2} L^{4}} .
$$

The Gaussian equivalent is

$$
\frac{1}{\tau_{j}^{(e f f)}}-\frac{1}{\tau_{j}^{(b)}}=\frac{4 \pi^{4} \ell^{2}}{L^{2}} \sum_{j^{\prime}=1}^{S} \frac{\left(a_{11}+a_{22}+2(-1)^{j+j^{\prime}} a_{12}\right) j^{2} j^{\prime 2} \tau_{j^{\prime}}^{(b)}}{\pi^{4}\left(j^{2}-j^{\prime 2}\right)^{2}\left(\tau_{j^{\prime}}^{(b)}\right)^{2}+m^{2} L^{4}} .
$$

When $R \gg \mathcal{L}_{b} \gg p_{0} L^{2} \sim S L$, the scattering-induced transitions between the minibands are suppressed, and Eq.(26) reduces to

$$
\frac{1}{\tau_{j}^{(e f f)}}-\frac{1}{\tau_{j}^{(b)}}=\frac{4 \pi^{4}}{m^{2} L^{6}}\left(\zeta_{11}(\mathbf{s}=0)+\zeta_{22}(\mathbf{s}=0)+2 \zeta_{12}(\mathbf{s}=0)\right) j^{4} \tau_{j}^{(b)},
$$

or, in the Gaussian case (27)

$$
\frac{1}{\tau_{j}^{(e f f)}}-\frac{1}{\tau_{j}^{(b)}}=\frac{4 \pi^{4} \ell^{2}}{m^{2} L^{6}}\left(a_{11}+a_{22}+2 a_{12}\right) j^{4} \tau_{j}^{(b)} .
$$

The non-Matthiessen's nature of these equations is obvious - the wall-driven term in the right hand side is strongly renormalized by and is directly proportional to the bulk relaxation time $\tau_{j}^{(b)}$. The value of $\chi_{j}$ is determined by the ratio of $1 / \tau_{j}^{(e f f)}-1 / \tau_{j}^{(b)}$, Eqs. $(28),(29)$, to the expressions for $1 / \tau_{j}^{(w)}$ from the previous subsection.

\section{E. Interference in ultrathin films}

In ultrathin films, $L \ll R, \mathcal{L}_{b}$, the distance between two peaks for $j \neq j^{\prime}$ is much larger than their width, and the integrals (15) vanish except for $j=j^{\prime}$. If $j=j^{\prime}$, both peaks are centered around the same value of $q_{j}=q_{j^{\prime}}$ and

$$
\frac{1}{\tau_{j}^{(e f f)}}-\frac{1}{\tau_{j}^{(b)}}=\frac{\pi^{3} j^{4} \tau_{j}^{(b)}}{m^{2} L^{6}} \int \frac{\zeta_{11}^{(0)}\left(q_{j}, q^{\prime}\right)+\zeta_{22}^{(0)}\left(q_{j}, q^{\prime}\right)+2 \zeta_{12}^{(0)}\left(q_{j}, q^{\prime}\right)}{\left(\epsilon_{j}\left(\mathbf{q}^{\prime}\right)-\mu\right)^{2}\left(2 \tau_{j}^{(b)}\right)^{2}+1} q^{\prime} d q^{\prime} .
$$

For Gaussian correlations, assuming that $q_{j} R \gg 1$ for all minibands $\epsilon_{j}$, direct integration yields 


$$
\begin{aligned}
\frac{1}{\tau_{j}^{(e f f)}}-\frac{1}{\tau_{j}^{(b)}} & =\frac{4 \pi^{9 / 2} l^{2} j^{4} \tau_{j}^{(b)}}{m^{2} L^{6}}\left(a_{11}+a_{22}+2 a_{12}\right) x_{j} \exp \left(x_{j}^{2}\right)\left(1-\operatorname{erf}\left(x_{j}\right)\right), \\
x_{j} & =\frac{R m}{2^{3 / 2} q_{j} \tau_{j}^{(b)}}, \operatorname{erf}(x)=\frac{2}{\sqrt{\pi}} \int_{0}^{x} \exp \left(-t^{2}\right) d t .
\end{aligned}
$$

When $L \ll R$, the condition $q_{j} R \gg 1$ can be violated only in the case of the single-band occupancy or, in multiband situations, for the highest miniband $q_{S}$.

\section{TRANSPORT TIME AND DIFFUSION COEFFICIENT}

Transport and localization parameters contain the transport time $\tau_{t r}$ rather than the effective relaxation time $\tau_{\text {eff }}$ of Section III. In quantized systems with $S$ occupied or energetically accessible minibands $\epsilon_{j}(\mathbf{q})$, the diffusion coefficient $D$ is expressed via $\tau_{t r}$ as

$$
D \equiv \frac{1}{2 m^{2}}\left\langle q^{2}\right\rangle \tau_{t r}=\frac{\tau_{t r}}{2 m^{2} S} \sum_{j=1}^{S} q_{j}^{2}, q_{j}=\left[2 m \mu-(\pi j / L)^{2}\right]^{1 / 2}
$$

Particle mobility (conductivity) can be easily obtained from Eq.(32) using the Einstein relation. The diffusion coefficient $D(E)$ and the mean free path $\mathcal{L}=2 D / v$ determine the localization length $\mathcal{R}$ for particles with energy $E[34,35,27]$

$$
\mathcal{R}(E)=\mathcal{L}(E) \exp [\varphi(E)], \varphi(E)=\pi m S(E) D(E)
$$

The transition from the relaxation time $\tau_{j}^{(e f f)}$ to the transport time $\tau_{t r}$ can be performed by solving the transport equation. Under usual circumstances, this cumbersome procedure results in a routine replacement $[25,24]$ of the zeroth angular harmonics of the scattering probabilities in expressions for $\tau$ by the difference of the zeroth and first harmonics, i.e., in addition of the transport factor $(1-\cos \theta)$ to the integrands. Our problem is more convoluted because of the matrix character of the equations and the angular dependence of $\tau$ in the denominators of the integrands (10). The proper diagrammatic procedure is described in Appendix $\mathrm{C}$ and is based on the realistic assumption that the bulk scattering vertex depends mainly on the angle between momenta of colliding particles. Even in this case, the solution of the matrix transport equation requires an inversion of large matrices 
of rank $S$. Since the transport equation contains an entangled combination of matrices that describe the interstate transitions caused by the bulk and boundary scattering, the inversion problem restricts analytical calculations of the transport time $\tau_{t r}$ in comparison with the effective collision time $\tau_{e f f}$ in Section III.

According to Appendix C, Eq.(63), the transport time can be expressed via the angular harmonics of the wall scattering probability $W(11)$ and bulk collision and transport times $\tau_{b}$ and $\tau_{t r}^{b}$ as

$$
\begin{aligned}
& \tau_{t r}=\sum_{j, j^{\prime}} q_{j} \Upsilon_{j j^{\prime}}^{-1} q_{j^{\prime}} / \sum_{j} q_{j}^{2}, \\
& \Upsilon_{j j^{\prime}}=\frac{\delta_{j j^{\prime}}}{\tau_{j}^{(e f f)}}-\frac{1}{\tau_{b}}\left[\frac{1}{\alpha S}+\int \frac{q^{\prime} d q^{\prime}}{4 \pi} \frac{W_{j j^{\prime}}^{(1)}\left(q_{j}, q^{\prime}\right)}{\left(\epsilon_{j^{\prime}}\left(q^{\prime}\right)-\mu\right)^{2}+1 / 4 \tau_{b}^{2}}\right] \text {, } \\
& \alpha=1+\frac{\tau_{b}}{\tau_{t r}^{b}-\tau_{b}} \frac{\left(\sum q_{j}\right)^{2}}{S \sum q_{j}^{2}} .
\end{aligned}
$$

Further calculation requires the inversion of the matrix $\Upsilon_{j j^{\prime}}$. Figures $4-6$ provide the numerical examples of the deviation $\chi_{t r}$ of the effective transport time from the one given by the Matthiessen's rule for independent bulk and boundary scattering,

$$
\chi_{t r} \equiv\left[\frac{1}{\tau_{t r}}-\frac{1}{\tau_{t r}^{b}}\right] /\left[\frac{1}{\tau_{t r}^{M}}-\frac{1}{\tau_{t r}^{b}}\right]
$$

where the "Matthiessen's" transport time $\tau_{t r}^{M}$ is determined by Eq.(34) but without bulkboundary interference,

$$
\tau_{t r}^{M}=\left[\sum_{j} q_{j}^{2}\right]^{-1} \sum_{j, j^{\prime}} q_{j}\left[\left(\frac{1}{\tau_{b}}+\frac{1}{\tau_{j}^{(w)}}\right) \delta_{j j^{\prime}}-\frac{1}{\alpha S \tau_{b}}-\frac{m}{2} W_{j j^{\prime}}^{(1)}\left(q_{j}, q_{j^{\prime}}\right)\right]^{-1} q_{j^{\prime}} .
$$

The Matthiessen's rule corresponds to $\chi_{t r}=1$. This definition of $\chi_{t r}$ is similar to the relative interference contributions $\chi_{j}$ to collision time, Eq.(16), in Section III. The difference $\chi_{t r}-1$ is the ratio of the interference contribution to the "pure" wall-driven transport time; when $\chi_{t r}-1>1$, as in Figure 6, the interference term dominates over the "pure" wall-driven contribution.

Figures $4-6$ illustrate this relative interference contribution $\chi_{t r}$. Figure 4 shows $\chi_{t r}$ as a function of the correlation radius of the surface inhomogeneities $p_{0} R$ for the inhomogeneities 
with the amplitude $p_{0} \ell=0.1$, bulk mean free path $p_{0} \mathcal{L}_{b}=10$, and the ratio of the bulk transport time to the bulk collision time $\tau_{t r}^{b} / \tau_{b}=1.5$. The three curves describe films of three different thicknesses: thin line $-p_{0} L=5$ (only the first miniband is energetically accessible); bold line $-p_{0} L=10$ (three minibands are accessible); dotted line $-p_{0} L=20$ (six minibands are accessible).

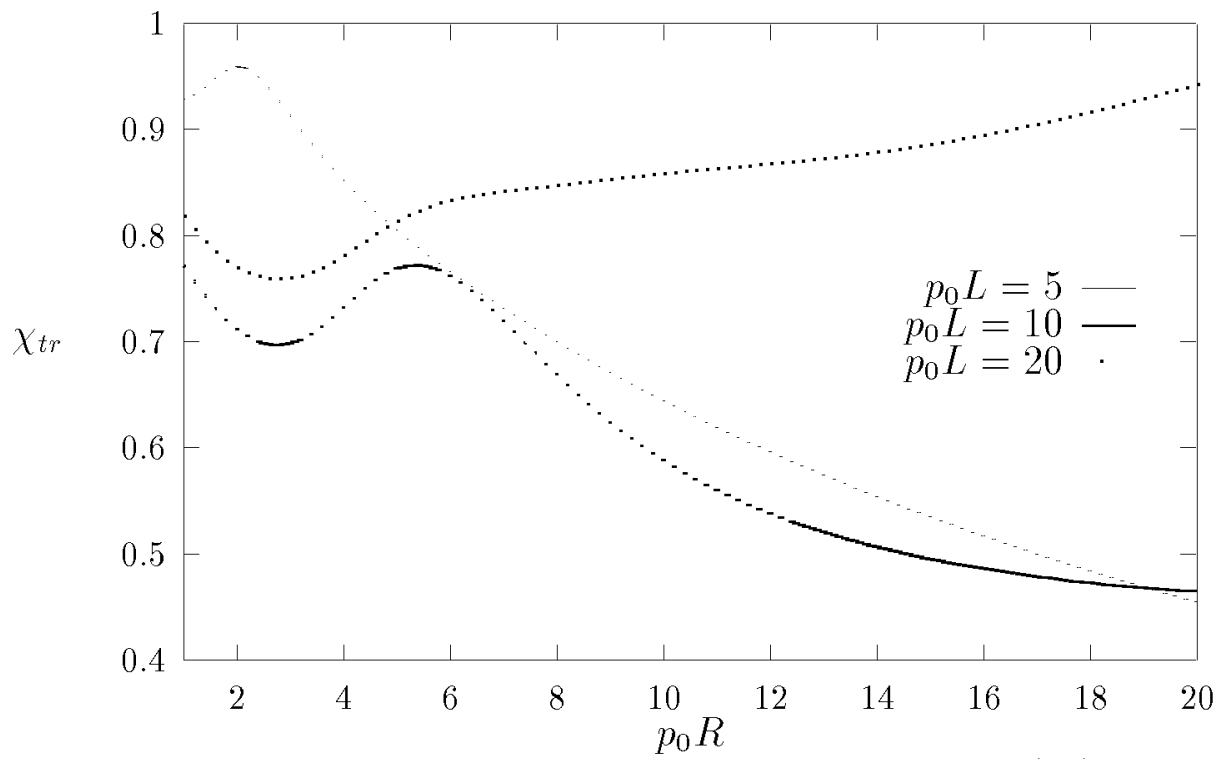

FIG. 4. Relative interference contribution to transport time, $\chi_{t r}$, Eq. (35), as a function of the size of the inhomogeneities $p_{0} R$ for $p_{0} \ell=0.1, p_{0} \mathcal{L}_{b}=10, \tau_{t r}^{b} / \tau_{b}=1.5$, and three values of the film thickness, $p_{0} L=5 ; 10 ; 20$.

Figure 5 presents $\chi_{t r}$ as a function of the bulk mean free path $p_{0} \mathcal{L}_{b}$ under the condition that the ratio of bulk transport and collision times does not change, $\tau_{t r}^{b} / \tau_{b}=1.5$. The amplitude of the inhomogeneities is $p_{0} \ell=0.1$, the surface correlation radius is $p_{0} R=10$, and the film is either $p_{0} L=5$ (thin line; only the first miniband is energetically accessible) or $p_{0} L=20$ (bold line; six minibands are accessible). With increasing bulk mean free path the interference effects disappear, $\chi \rightarrow 1$. 


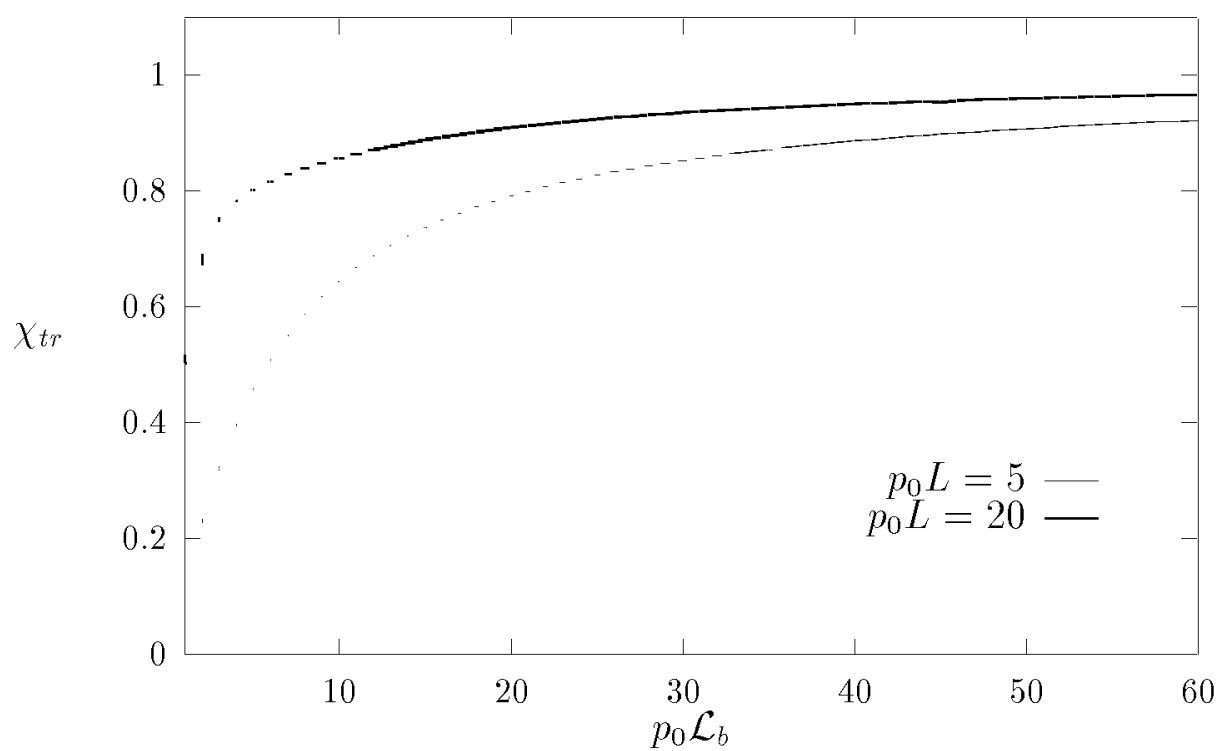

FIG. 5. Relative interference contribution to transport time, $\chi_{t r}$, Eq. (35), as a function of the bulk free path $p_{0} \mathcal{L}_{b}$ at constant ratio $\tau_{t r}^{b} / \tau_{b}=1.5$. The inhomogeneities are characterized by $p_{0} R=10, p_{0} \ell=0.1 ;$ the film thickness is $p_{0} L=5 ; 20$.

Figure 6 shows $\chi_{t r}$ as a function of the film thickness $p_{0} L$ for the inhomogeneities with the amplitude $p_{0} \ell=0.1$, bulk mean free path $p_{0} \mathcal{L}_{b}=20$, and the ratio of the bulk transport and collision times $\tau_{t r}^{b} / \tau_{b}=1.5$. The two curves correspond to two different correlation radii of the surface inhomogeneities: thin line $-p_{0} R=50$; bold line $-p_{0} R=20$. The singularities reflect to the change in number of energetically accessible minibands with increasing film thickness. 


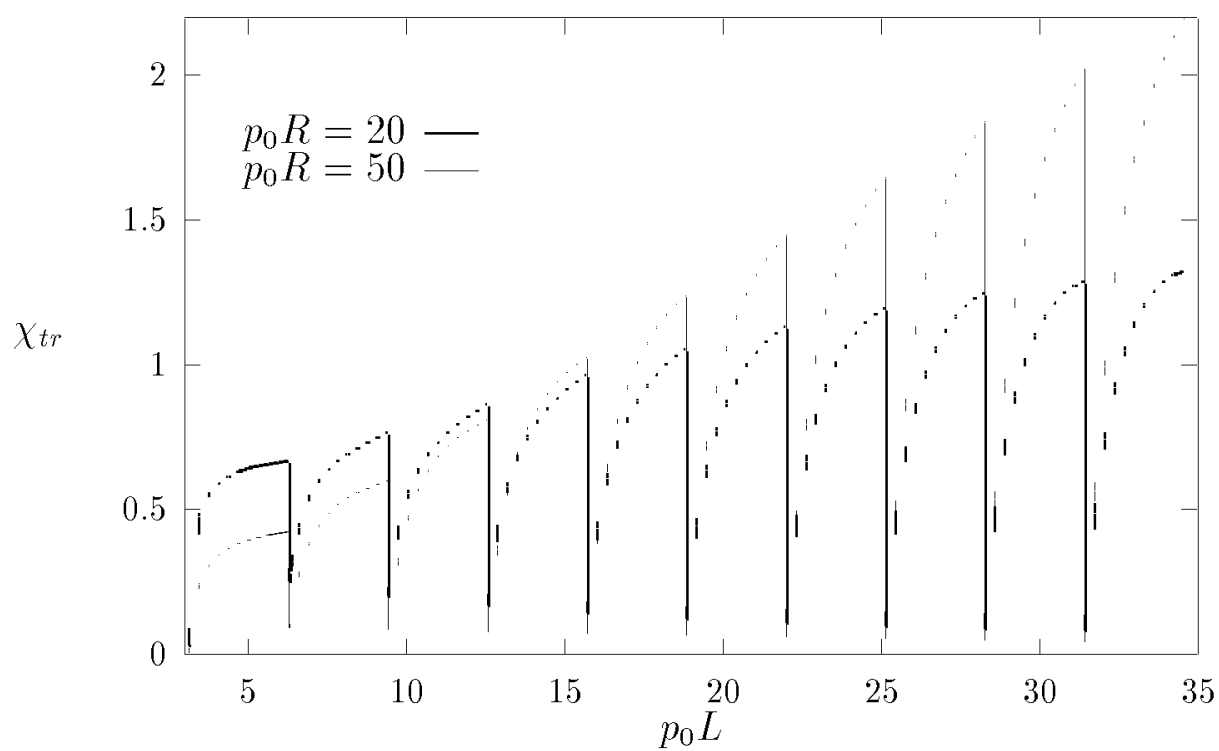

FIG. 6. Relative interference contribution to transport time, $\chi_{t r}$, Eq. (35), as a function of the film thickness $p_{0} L$ for $p_{0} \ell=0.1, p_{0} \mathcal{L}_{b}=20, \tau_{t r}^{b} / \tau_{b}=1.5$, and two values of the correlation radius, $p_{0} R=20 ; 50$.

Occasionally, the interference contribution to the transport time can be calculated analytically.

\section{A. Single-band systems}

Inversion is a non-issue for single-band systems. Physically, the single-band situation corresponds either to systems with one quantized state $\epsilon_{1}(\mathbf{q})=\epsilon_{1}+q^{2} / 2 m$, such as for particles adsorbed on or bound to the wall, or to multiband systems in which the gaps between the minibands are large in comparison to the particle energy, $E \lesssim 1 / m L^{2}$, and only the first miniband is energetically accessible.

For single-band particles, Eq.(34) yields

$$
\frac{1}{\tau_{t r}}=\frac{1}{\tau_{t r}^{b}}+\frac{1}{2 \tau_{b}} \int \frac{q^{\prime} d q^{\prime}}{2 \pi} \frac{W_{11}^{(0)}\left(q_{1}, q^{\prime}\right)-W_{11}^{(1)}\left(q_{1}, q^{\prime}\right)}{\left(\epsilon_{1}\left(q^{\prime}\right)-\mu\right)^{2}+1 / 4 \tau_{b}^{2}} .
$$

This integral can be calculated explicitly in the same limiting cases as in Section III.

Large bulk mean free path. If the bulk mean free path is large, $\mathcal{L}_{b} \gg R$, the denominator behaves effectively as the $\delta$-function (19) and 


$$
\begin{aligned}
\frac{1}{\tau_{t r}} & =\frac{1}{\tau_{t r}^{b}}+\frac{m}{2}\left[W_{11}^{(0)}\left(q_{1}, q_{1}\right)-W_{11}^{(1)}\left(q_{1}, q_{1}\right)\right] \\
& =\frac{1}{\tau_{t r}^{b}}+\frac{2 \pi^{5} \ell^{2} R^{2}}{m L^{6}}\left(a_{11}+a_{22}+2 a_{12}\right){ }_{1} F_{1}\left(\frac{3}{2}, 2,-2 q_{1}^{2} R^{2}\right),
\end{aligned}
$$

where the last expression was calculated for the Gaussian correlation of inhomogeneities.

Small bulk mean free path. In the opposite cas, $\mathcal{L}_{b} \ll R$, the numerator in the integrand (37) is a narrow peak and

$$
\begin{aligned}
\frac{1}{\tau_{t r}} & =\frac{1}{\tau_{t r}^{b}}+\frac{\tau_{b}}{\pi^{2}} \int W_{11}\left(\mathbf{q}_{1}-\mathbf{q}^{\prime}\right)(1-\cos \theta) d \mathbf{q}^{\prime} \\
& =\frac{1}{\tau_{t r}^{b}}+\frac{2 \pi^{4} \ell^{2} \tau_{b}}{m^{2} L^{6} q_{1}^{2} R^{2}}\left(a_{11}+a_{22}+2 a_{12}\right),
\end{aligned}
$$

where in the calculation of the integral for the Gaussian correlation function it was important that $q_{1} R \gg 1$.

Short-wave particles. If the particle momentum is large, $q_{1} R \gg 1$, the integral (37) for the Gaussian correlation function yields the result similar to Eq.(31),

$$
\frac{1}{\tau_{t r}}=\frac{1}{\tau_{t r}^{b}}+\frac{\pi^{9 / 2} \ell^{2}}{2^{1 / 2} q_{1}^{3} m R L^{6}}\left(a_{11}+a_{22}+2 a_{12}\right) \exp \left(x_{1}^{2}\right)\left(1-\operatorname{erf}\left(x_{1}\right)\right) .
$$

\section{B. Ultrathin films}

Calculations can also be performed analytically for ultrathin films, $L \ll R, \mathcal{L}_{b}$. Though in this case all the matrices can be inverted analytically, the general expressions are too cumbersome to be given here. The complications arise from the fact matrix $\Upsilon_{j j^{\prime}}$ in Eq.(34) consists of the sum of the term with the diagonal matrix $W_{j j^{\prime}}$ and the index-independent term $1 / \alpha_{1} S$. If the correlation function is Gaussian, the resulting transport time is

$$
\begin{aligned}
\frac{1}{\tau_{t r}} & =\left[\sum q_{j}^{2}\right] /\left[\sum \frac{q_{j}^{2}}{Q_{j}}+\frac{\left[\sum q_{j} / Q_{j}\right]^{2}}{\alpha_{1} S \tau_{b}-\sum Q_{j}^{-1}}\right] \\
Q_{j} & =\frac{1}{\tau_{b}}+\frac{\pi^{9 / 2} \ell^{2}}{2^{1 / 2} q_{j}^{3} m R L^{6}}\left(a_{11}+a_{22}+2 a_{12}\right) \exp \left(x_{j}^{2}\right)\left(1-\operatorname{erf}\left(x_{j}\right)\right) .
\end{aligned}
$$




\section{Long-wave particles}

The last analytical case is the case of long-wave particles $p_{0} R \ll 1$, when all the scattering probabilities are constant, and the transport time has the same structure as Eq.(41) with

$$
Q_{j}=\frac{1}{\tau_{b}}+\frac{\pi^{4} j^{2}}{6 m L^{6}} S(S+1)(2 S+1)\left[\zeta_{11}(0)+\zeta_{22}(0)+\frac{6(-1)^{S+j}}{2 S+1} \zeta_{12}(0)\right] .
$$

(in this case, all $W_{j j^{\prime}}^{(0)}=2 W(0), W^{(1)}=0$ and the inversion is similar). In the Gaussian case, $\zeta_{i k}(q=0)$ should be replaced by $2 \pi a_{i k} \ell^{2} R^{2}$.

\section{SUMMARY}

In summary, we calculated the effective collision and transport times in ultrathin quantized systems with boundary and bulk scattering. The results describe transport and localization in ultrathin films with QSE. Scattering by the surface inhomogeneities is strongly renormalized by bulk scattering processes which are responsible for the repeated returns of particles to the walls. With the exception of the nearly ballistic regime, strong interference of bulk and boundary scattering invalidates description of these two scattering channels as independent relaxation processes. Under certain conditions, the interference contribution to transport can even exceed the "pure" wall term.

Exact results require the information on the bulk scattering vertex in quantized films which is not always available. A more technical difficulty is the matrix nature of equations for quantized systems. Often, these two issues can be accurately resolved. Elsewhere, it is possible to introduce reasonable approximations. The main approximation - the form of the bulk scattering vertex - is not germane to the main goal of this paper, namely, to the incorporation of boundary scattering into the bulk transport theory.

The effective collision and transport times are expressed explicitly via the bulk relaxation times and statistical parameters of the surface corrugation. Under certain conditions, such as for ultrathin system, nearly ballistic particles, and for robust bulk scattering, the analytical expressions for the effective time are quite simple and can be used without specifying the form 
of the surface correlation function. Elsewhere, the effective time is calculated numerically for the Gaussian correlation of surface inhomogeneities.

To avoid parameter clutter, the numerical examples are given for the simplest singlelayer films. The extension of the results to multilayer films, non-degenerate semiconductors, non-uniform internal potentials, etc., is straightforward and can be done in the same way as in Ref. [27] for purely ballistic quantized films with corrugated surfaces.

Our quantum results on boundary - bulk interference are simpler, except for the quantum resonance region [26], than the quasiclassical integral equations. In the semi-ballistic limit, the quantum results are preferable even for relatively thick films: In contrast to the quasiclassical picture, the quantum approach includes the interference between particles scattered by different walls. It also eliminates the divergencies, which are inherent to quasiclassical ballistic systems and are caused by a disproportional contribution from particles with momenta directed along the walls.

The main conclusion is that the transport calculations for quantized films, performed with independent bulk and wall scattering, are often wrong. These results should and can be modified so that to include interference.

\section{ACKNOWLEDGMENTS}

The work was supported by NSF grant DMR-9705304. 


\section{REFERENCES}

[1] K.L.Chopra, Thin Film Phenomena (McGrow Hill, NY) 1969

[2] V.I.Okulov, and V.V.Ustinov, Sov.J.Low Temp.Phys. 5, 101 (1979)

[3] T.Ando, A.B.Fowler, and F.Stern, Rev.Mod.Phys. 54, 437 (1982)

[4] V.B.Sandomirskii, Sov.Phys.-JETP 25, 101 (1967) [Zh.Eksp.\&Teor.Fiz. 52, 158 (1968)]

[5] N.Trivedi, and N.W.Ashcroft, Phys.Rev. B 38, 12298 (1988)

[6] G.Fishman, and D.Calecki, Phys.Rev.Lett., 62, 1302 (1989); Phys.Rev. B 43, 11581 (1991)

[7] A.Kawabata, J.Phys.Soc.Jap., 62, 3988 (1993)

[8] N.M.Makarov, A.V.Moroz, and V.A.Yampol'skii, Phys.Rev. B 52, 6087 (1995)

[9] A.E.Meyerovich, and S.Stepaniants, J.Phys.:Cond.Matt. 9, 4157 (1997)

[10] G.Palasantzas, and J.Barnas, Phys.Rev. B 56, 7726 (1997)

[11] L. A. Kuzik, Yu. E. Petrov, F. A. Pudonin, and V. A. Yakovlev, Sov.Phys. - JETP 78, 114 (1994) [Zh.Exp.Teor.Fiz. 105, 215 (1994)]

[12] P.A.Badoz, $\quad$ A.Briggs, E.Rosencher, A.A.d'Avitaya, and C.d'Anterroches, Appl.Phys.Lett. 51, 169 (1985); J.Y.Duboz, P.A.Badoz, E.Rosencher, J.Henz, M.Ospelt, H. von Kanel, and A.Briggs, Appl.Phys.Lett. 53,788 (1988); M.Jalohowski, E.Bauer, H.Knoppe, and G.Lilienkamp, Phys.Rev. B 45, 13607 (1992);; A. Carl, G. Dumpich, and E. F. Wassermann, Phys.Rev. B 50, 4802 (1994); M.Jalohowski, H.Hoffman, and E.Bauer, Phys.Rev.Lett. 76, 4227 (1996); G.M.Mikhailov, I.V.Malikov, and A.V.Chernykh, JETP Lett. 66, 725 (1997) [Pis'ma Zh. Eksp. Teor. Fiz. 66, 603 (1997)]

[13] H.Sakaki, T. Noda, K. Hirakawa, M. Tanaka, and T. Matsusue, Appl.Phys.Lett. 51, 
1934 (1987); A. Gold et al, Solid State Comm. 60, 531 (1986); L.-W.Tu, G.K.Wong, and J.B.Ketterson, Appl.Phys.Lett. 55, 1327 (1989); J.Liu, and L.Feng, Appl.Phys.Lett. 63, 2783 (1993); L. Bolotov, T. Tsuchiya, A. Nakamura, T. Ito, Y. Fujiwara, and Y. Takeda, Phys.Rev. B 59, 12236 (1999)

[14] G.Reiss, E.Hastreiter, H.Bruckl, and J.Vancea, Phys.Rev. B 43, 5176 (1991); I. B. Altfeder, K. A. Matveev, and D. M. Chen, Phys.Rev.Lett. 78, 2815 (1997); I. B. Altfeder, D. M. Chen, and K. A. Matveev, Phys.Rev.Lett. 80, 4895 (1998); L. Bolotov, T. Tsuchiya, A. Nakamura, T. Ito, Y. Fujiwara, and Y. Takeda, Phys.Rev. B 59, 12236 (1999)

[15] M.Jalohowski, H.Knoppe, G.Lilienkamp, and E.Bauer, Phys.Rev. B 46, 4693 (1992); J.E.Ortega, F.J.Himpsel, G.J.Mankey, and R.F.Willis, Phys.Rev. B 47, 15401 (1993); I. Sagnes, A. Halimaoui, G.Vincent, and P. A. Badoz, Appl.Phys.Lett. 62, 1155 (1993); S. Tong, X. Liu, T. Gao, X. Bao, Appl.Phys.Lett. 71, 698 (1997)

[16] S.L. Phillipson, A.M. Guenault, S.N. Fisher, G.R.Pickett, and P.J.Y. Thibault, Nature 395, 578 (1998).

[17] H. Moraal, Phys.Rep. 17, 225 (1975)

[18] P.M.Levy, S.Zhang, and A.Fert, Phys.Rev.Lett. 65, 1643 (1990)

[19] D.Calecki, Phys.Rev. B 42, 6906 (1990)

[20] V. L. Gurevich, V. B. Pevzner, and K. Hess, Phys.Rev. B 51, 5219 (1995)

[21] L.Sheng, D.Y.Xing, and Z.D.Wang, Phys.Rev. B 51, 7325 (1995)

[22] M.K.Schwalm, and W.A.Schwalm, Phys.Rev. B 45, 6868 (1990)

[23] K.Nikolić, and A.MacKinnon, Phys.Rev. B 50, 11008 (1994)

[24] A.A.Abrikosov, L.P.Gorkov, and I.E.Dzyaloshinski, Methods of Quantum Field Theory in Statistical Physics (Dover, NY) 1975, Ch. 7 
[25] J.Rammer, and H.Smith, Rev.Mod.Phys., 58, 323 (1986)

[26] A. E. Meyerovich, and A. Stepaniants, Phys.Rev. B 58, 13242 (1998)

[27] A. E. Meyerovich, and A. Stepaniants, Phys.Rev. B 60, 9129 (1999)

[28] Z.Tesanovic, M.V.Jaric, and S.Maekawa, Phys.Rev.Lett. 57, 2760 (1986)

[29] A.E.Meyerovich, and S.Stepaniants, Phys.Rev.Lett. 73, 316 (1994); Phys.Rev. B 51, $17116(1995)$

[30] A.M.Bratkovsky, and S.N.Rashkeev, Phys.Rev. B 53, 13074 (1996)

[31] A. M. Bratkovsky, A. P. Sutton, and T. N. Todorov, Phys.Rev. B 52, 5036 (1995)

[32] J.A.Ogilvy, Theory of Wave Scattering from Random Surfaces (Adam Hilger, Bristol) 1991

[33] R.M.Feenstra et al, Phys.Rev.Lett. 72, 2749 (1994)

[34] P.A.Lee, and T.V.Ramakrishnan, Rev.Mod.Phys., 57, 287 (1985)

[35] A.McGurn, and A.Maradudin, Phys.Rev. B 30, 3136 (1984)

\section{APPENDIX A. DIAGRAMS FOR THE SELF-ENERGY AND RELAXATION TIME}

Self-energy diagrams are built of the free-particle Green's functions and three types of interaction, $\widehat{V}, \delta U$, and $U$, Eq.(9), following the usual rules of the diagrammatic technique. The volume averaging over the distribution of impurities and the averaging over the surface inhomogeneities can be done using the standard method [24-26]. The result should contain the full summation over the impurity diagrams, but be only of the second order in surface inhomogeneities. This means that the relevant diagrams include $U$ in all possible configurations, but only two vertices corresponding to either $\widehat{V}$ or $\delta U$.

Without boundary scattering, the integral equation of Figure 7 a expresses the (retarded) 
$G$-function with bulk impurity scattering $G^{(i)}$ (bold line) via the free-particle Green's function $G^{(0)}$ (thin line) and the interaction with impurities $U$ (cross) in all orders.

The addition of the perturbation $\widehat{V}$ results in the diagram of Figure $7 b$ in which the shaded line is the Green's function $G$ for the perturbation $\widehat{V}+U$, the bold line is $G^{(i)}$, and the star is $\hat{V}$. Since the diagrams with an odd number of the "stars" $\hat{V}$ vanish after averaging over the surface inhomogeneities $\xi$, the diagrammatic equation in Figure $7 b$ is equivalent to the one in Figure $7 c$.

Averaging $[25,24,26]$ is done by connecting of crosses and stars between themselves (by dashed lines, as in Figure 8). The wall inhomogeneities are assumed to be small and one should consider the diagrams with no more than two stars. For impurities we perform the full summation beyond the Born approximation and, therefore, take into account in $\left\langle G^{(i)}\right\rangle_{V}$ the multicross diagrams such as, for example, in Figure $8 a, b$.

When $p_{0} \mathcal{L} \gg 1$, scattering does not renormalize the particle energy and results only in the formation of the mean free path and relaxation time. The same condition allows one to disregard all the diagrams with the intersecting dashed lines, as the ones in Figure $8 b, c$, in comparison with the ones with the non-intersecting lines and multiline connections such as in Figure $8 a$ [24]. The explanation is that the angular integration for the intersecting diagrams gets restricted to a small solid angle irrespective of whether the intersecting lines have the same interaction sources, as crosses in Figure $8 b$, or different sources, as crosses and stars in Figure $8 c$.

The remaining diagrams contain one dashed star line $(\widehat{V}-\widehat{V})$ with all possible impurity (cross) lines either above or below, but not intersecting it. The summation of all impurity lines below the $\widehat{V}-\hat{V}$ line yields immediately the bold impurity line $\left\langle G^{(i)}\right\rangle_{V}$. Then the sum of all remaining diagrams with the non-intersecting impurity lines outside and above the $\hat{V}-\hat{V}$ (star-star) line leads to the equation of Figure 9 for the self-energy function $\Sigma$ where $\Gamma$ is the full bulk (impurity) scattering vertex.

Here, the first term in the right hand side is the self-energy with bulk impurities and 
without any boundary scattering,

$$
\operatorname{Im} \Sigma_{1}^{R}=-\frac{1}{2 \tau_{j}^{(b)}(\mathbf{q})}
$$

The second term is the sum of all diagrams in which the wall scattering line $\widehat{V}-\widehat{V}$ is the outside line,

$$
\operatorname{Im} \Sigma_{2}^{R}=\operatorname{Im} \sum_{j^{\prime}=1}^{S} \int \frac{W_{j j^{\prime}}\left(\mathbf{q}, \mathbf{q}^{\prime}\right)}{\omega-\epsilon_{j^{\prime}}\left(\mathbf{q}^{\prime}\right)+\mu+i / 2 \tau_{j^{\prime}}^{(b)}\left(\mathbf{q}^{\prime}\right)} \frac{d \mathbf{q}^{\prime}}{(2 \pi)^{2}},
$$

with the wall-induced transition probability $W_{j j^{\prime}}\left(\mathbf{q}, \mathbf{q}^{\prime}\right)$ from Ref. [26]:

$$
W_{j j^{\prime}}\left(\mathbf{q}, \mathbf{q}^{\prime}\right)=\frac{\pi^{4}}{m^{2} L^{6}}\left(\zeta_{11}\left(\mathbf{q}-\mathbf{q}^{\prime}\right)+\zeta_{22}\left(\mathbf{q}-\mathbf{q}^{\prime}\right)+2(-1)^{j+j^{\prime}} \zeta_{12}\left(\mathbf{q}-\mathbf{q}^{\prime}\right)\right) j^{2} j^{\prime 2}
$$

The last diagram includes the diagrams with impurity lines both above and below the $\widehat{V}-\hat{V}$ line and is very complicated. If $p_{0} \mathcal{L}_{b} \gg 1$, the full vertex $\Gamma$ does not differ from the irreducible one, $\Gamma \approx \widetilde{\Gamma}$. This latter vertex in the momentum space changes on a large scale $1 / r_{0}$ (the interaction radius $r_{0}$ is often small, Eq.(7)). The zeroth angular harmonic of the irreducible impurity vertex gives the impurity relaxation time,

$$
\frac{1}{\tau_{j}^{(b)}}=\frac{m}{2} \sum_{j^{\prime}} \widetilde{\Gamma}_{j j^{\prime}}^{(0)}
$$

Direct calculation of the diagram for slowly varying (almost constant) vertex shows that the contribution of this diagram is negligible in comparison with (44) when $p_{0} \mathcal{L}_{b} \gg 1$. If the inequality $(7)$ does not hold $-r_{0}$ is not small or $p_{0} \mathcal{L}_{b}$ is not large - the computation of this third term requires, as an input, an accurate model for the impurity vertex $\widetilde{\Gamma}_{j j^{\prime}}\left(\mathbf{q}, \mathbf{q}^{\prime}\right)$.

When the conditions (7) are met, the last diagram in Figure 9 can be disregarded. As a result, the averaging over impurities, $\langle\ldots\rangle_{V}$ and surface inhomogeneities $\langle\ldots\rangle_{\xi}$, reduces the effective relaxation time (the imaginary part of the self energy, $\Sigma^{A}-\Sigma^{R}$ ) to

$$
\frac{1}{\tau_{j}^{(e f f)}(\mathbf{q})}=\frac{1}{\tau_{j}^{(b)}(\mathbf{q})}+\sum_{j^{\prime}=1}^{S} \int \frac{W_{j j^{\prime}}\left(\mathbf{q}, \mathbf{q}^{\prime}\right) / \tau_{j^{\prime}}^{(b)}\left(\mathbf{q}^{\prime}\right)}{\left(\epsilon_{j^{\prime}}\left(\mathbf{q}^{\prime}\right)-\mu\right)^{2}+\left(1 / 2 \tau_{j^{\prime}}^{(b)}\left(\mathbf{q}^{\prime}\right)\right)^{2}} \frac{d \mathbf{q}^{\prime}}{(2 \pi)^{2}}
$$

It is clear that if one operates in terms of the relaxation time $\tau_{j}^{(b)}(\mathbf{q})$ without specifying its form, there is no difference between impurity scattering and other bulk scattering mechanisms, such as particle-particle collisions, as long as the interaction potential is sufficiently short-range. 
As for the diagrams with the interaction $\delta U$, lengthy analysis shows that these diagrams can be disregarded when

$$
p_{0} \mathcal{L}_{b} \gg L^{2} / j^{2} S^{2} R^{2}, 1
$$

In this paper, we do not consider the anomalous region in which $\delta U$ is important though in this region the deviation from the Matthiessen's rule (1) is more profound than elsewhere. The only physical situation in which one can observe these anomalous effects is $L / S \ll \mathcal{L}_{b} \ll$ $\left(L / S j^{2}\right)\left(L^{2} / S^{2} R^{2}\right)$ at $L^{2} / S^{2} R^{2} \gg j^{2}$. Since the wall contribution can be observed only for not very small bulk free paths, $\mathcal{L}_{b} \gtrsim L$, this condition is too restrictive and the anomalous region, in which the diagrams with $\delta U$ prevail, is narrow. Though the wall contribution in the anomalous region is very unusual and its interference with the bulk terms is large, the chance to observe this ultra-quantum situation is rather slim, at least for electrons. For long-wave photons or phonons the chances are higher.

\section{APPENDIX B. BULK RELAXATION TIMES IN QUANTIZED FILMS}

The explicit form of the volume average of the Green's function with impurity scattering $\left\langle G^{(i)}\right\rangle_{V}$ in thin films with QSE differs from its bulk analog [24] even for perfect walls. This complicates the evaluation of the relaxation times $\tau_{j}^{(b)}(\mathbf{q})$ in quantized minibands even when the whole function $\tau_{b}(\mathbf{p})$ in unrestricted bulk is known. We will give the expressions for $\tau_{j}^{(b)}(\mathbf{q})$ in the ladder (Born) approximation. As above, one can disregard the diagrams with the intersecting lines such as in Figure $8 b$ if $p_{0} \mathcal{L} \gg 1$, and the only important diagrams not taken into account by the ladder series are the multiline connections such as the ones in Figure 8 a. Standard ladder calculation for a thin film with perfect walls yields

$$
i / \tau_{j}^{(b)}(\mathbf{q})=-\frac{N_{i m p}}{L} \sum_{j^{\prime}} \int \frac{d \mathbf{q}^{\prime}}{(2 \pi)^{2}} \frac{\left|u_{j^{\prime}+j}\left(\mathbf{q}^{\prime}-\mathbf{q}\right)\right|^{2}+\left(1+\delta_{j j^{\prime}}\right)\left|u_{j^{\prime}-j}\left(\mathbf{q}^{\prime}-\mathbf{q}\right)\right|^{2}}{-\epsilon_{j^{\prime}}\left(\mathbf{q}^{\prime}\right)+\mu+i 0} .
$$

The integral in Eq.(49) contains the imaginary part (relaxation), and the real part which is responsible for the line shift (mean field). The real part is small in parameter $1 / p_{0} \mathcal{L} \ll 1$, the peak is narrow, and 


$$
\frac{1}{\tau_{j}^{(b)}(\mathbf{q})}=\frac{\pi N_{i m p}}{L} \sum_{j^{\prime}} \int \frac{d \mathbf{q}^{\prime}}{(2 \pi)^{2}} \delta\left(\epsilon_{j^{\prime}}\left(\mathbf{q}^{\prime}\right)-\mu\right)\left(\left|u_{j^{\prime}+j}\left(\mathbf{q}^{\prime}-\mathbf{q}\right)\right|^{2}+\left(1+\delta_{j j^{\prime}}\right)\left|u_{j^{\prime}-j}\left(\mathbf{q}^{\prime}-\mathbf{q}\right)\right|^{2}\right) .
$$

The transition to the standard expressions for unrestricted bulk geometry is simple. In thick quasiclassical films with high quantum numbers $j \gg 1$ the summation in Eq.(50) can be replaced by the integration over $p_{x}=\pi j / L$ from 0 to $\infty$. The transformation $p_{x} \rightarrow-p_{x}$ in the second term in the integrand allows one to rewrite the integral as a single integral from $-\infty$ to $\infty$ and reduces it to

$$
\frac{1}{\tau_{b}(\mathbf{p})}=N_{i m p} \frac{p_{0} m}{(2 \pi)^{2}} \int\left|u\left(\left|\mathbf{p}_{0}-\mathbf{p}\right|\right)\right|^{2} d \Omega
$$

where $p_{0}=(2 m \mu)^{1 / 2}$, and $\Omega$ is the solid angle between $\mathbf{p}$ and $\mathbf{p}_{0}$. The interaction range $r_{0}$ is short, Eq.(7), and in $u\left(\left|\mathbf{p}_{0}-\mathbf{p}\right|\right)$ one can replace $|\mathbf{p}|$ by $p_{0}$ making $\tau_{b}$ a momentumindependent constant. In metals, this equation reduces to a standard bulk expression for the relaxation time on the Fermi surface,

$$
\frac{1}{\tau_{b}}=N_{i m p} \frac{p_{F} m}{2 \pi} \int_{-1}^{1}\left|u\left(p_{F}(1-\cos \theta)\right)\right|^{2} d \cos \theta .
$$

For the same reason, the dependence of $\tau_{j}^{(b)}(\mathbf{q})$ on $\mathbf{q}$ in Eq. $(50)$ is very slow, and the relaxation times $\tau_{j}^{(b)}$ can be considered constant,

$$
\tau_{j}^{(b)}(\mathbf{q}) \simeq \tau_{j}^{(b)}\left(q_{j}\right) \equiv \tau_{j}^{(b)}
$$

\section{APPENDIX C. TRANSPORT TIME}

In transport phenomena, the observable is the transport time $\tau_{t r}$ rather than the collision $\tau$ of Appendix A. Under the usual circumstances, the difference between these two times reduces to a factor $(1-\cos \theta)$ in the integrands responsible for the angular averaging. In our

case, this is not so because of, first, the quantization of motion and, second, the presence of two scattering mechanisms of different nature, $\widehat{U}$ and $\hat{V}$. 
In bulk transport theory, the diagrams for the two-particle Green's function (or, after one integration, for the density propagator $P(\mathbf{p} ; \omega, \mathbf{k}))$ reduce to the equation [25]

$$
\begin{aligned}
(\omega-\mathbf{k} \cdot \mathbf{p} / m+i / \tau) P(\mathbf{p} ; \omega, \mathbf{k})= & {\left[G^{A}(0 ; \mathbf{p})-G^{R}(\omega ; \mathbf{p}+\mathbf{k})\right] \times } \\
& {\left[1+\int \frac{d \mathbf{p}^{\prime}}{(2 \pi)^{d}} \widetilde{\Gamma}\left(\mathbf{p}, \mathbf{p}^{\prime} ; \omega, \mathbf{k}\right) P\left(\mathbf{p}^{\prime} ; \omega, \mathbf{k}\right)\right] }
\end{aligned}
$$

Integration leads to the cooperon diagram and, in the end, defines the diffusion coefficient $D$ (or the transport time $\tau_{t r}$ ) as a pole in the density response function,

$$
\int \frac{d \mathbf{p}}{(2 \pi)^{d}} P(\mathbf{p} ; \omega, \mathbf{k}) \propto\left[-i \omega+D(\omega, k) k^{2}\right]^{-1},
$$

where, normally, one should consider $\omega, k \rightarrow 0$ in the argument of $D$.

In quantized films with two types of scattering, Eq.(54) has the matrix form

$$
\begin{aligned}
\left(\omega-\frac{\mathbf{k} \cdot \mathbf{q}}{m}+\frac{i}{\tau_{j}^{(e f f)}}\right) P_{j}(\mathbf{q} ; \omega, \mathbf{k})= & {\left[G_{j}^{(i) A}(\mathbf{q})-G_{j}^{(i) R}(\mathbf{q})\right] \times } \\
& {\left[1+\sum_{j^{\prime}} \int \frac{d \mathbf{q}^{\prime}}{(2 \pi)^{2}}\left(\widetilde{\Gamma}_{j j^{\prime}}\left(\mathbf{q}, \mathbf{q}^{\prime}\right)+W_{j j^{\prime}}\left(\mathbf{q}, \mathbf{q}^{\prime}\right)\right) P_{j^{\prime}}\left(\mathbf{q}^{\prime} ; \omega, \mathbf{k}\right)\right], }
\end{aligned}
$$

where $G_{j}^{(i)}$ are the Green's functions with impurity scattering and $\omega=k=0$ in all appropriate places. Then the matrix equation for the density response function becomes

$$
\sum_{j^{\prime}} \Theta_{j j^{\prime}} \int \frac{d \mathbf{q}}{(2 \pi)^{2}} P_{j^{\prime}}(\mathbf{q} ; \omega, \mathbf{k})=m
$$

where the effective frequencies $\Theta$ are defined as

$$
\begin{aligned}
\Theta_{j j^{\prime}} & =-i \omega \delta_{j j^{\prime}}+\Upsilon_{j j^{\prime}}^{(0)}+k^{2} \frac{q_{j} q_{j^{\prime}}}{2 m^{2}} \Upsilon_{j j^{\prime}}^{(1)-1}, \\
\Upsilon_{j j^{\prime}}\left(q_{j}, q_{j^{\prime}}, \cos \theta\right) & =\frac{\delta_{j j^{\prime}}}{\tau_{j}^{(e f f)}}-\frac{m}{2} \widetilde{\Gamma}_{j j^{\prime}}\left(q_{j}, q_{j^{\prime}}, \cos \theta\right)-\int \frac{q^{\prime} d q^{\prime}}{4 \pi} \frac{W_{j j^{\prime}}\left(q_{j}, q^{\prime}, \cos \theta\right) / \tau_{j^{\prime}}^{(b)}\left(\mathbf{q}^{\prime}\right)}{\left(\epsilon_{j^{\prime}}\left(\mathbf{q}^{\prime}\right)-\mu\right)^{2}+\left(1 / 2 \tau_{j^{\prime}}^{(b)}\left(\mathbf{q}^{\prime}\right)\right)^{2}},
\end{aligned}
$$

$\Upsilon_{j j^{\prime}}^{(0,1)}$ are the zeroth and first angular harmonics of the functions $\Upsilon_{j j^{\prime}}(\cos \theta)$ over the angle $\widehat{\mathbf{q q}^{\prime}}$, and $1 / \tau_{j}^{(e f f)}$ is given by Eq. (47). The Ward identity ensures that

$$
\frac{1}{\tau_{j}^{(e f f)}}=\frac{m}{2} \sum_{j^{\prime}} \widetilde{\Gamma}_{j j^{\prime}}^{(0)} .
$$

The diffusion coefficient can be calculated either from the matrix $\Theta_{j j^{\prime}}$ 


$$
\sum_{j, j^{\prime}} \Theta_{j j^{\prime}} \propto-i \omega+D(\omega, k) k^{2}
$$

or, alternatively, from the kinetic (transport) equation for the first angular harmonic $n_{j}^{(1)}$ of the distribution function $\delta n_{j}(\mathbf{q} ; \omega, \mathbf{k})$ of particles in the miniband $j$ :

$$
\left(\frac{d n_{j}}{d t}\right)^{(1)}=-\sum_{j^{\prime}} \Upsilon_{j j^{\prime}}^{(1)} n_{j^{\prime}}^{(1)}
$$

with the help of the diffusion current

$$
\mathbf{J}=\frac{1}{m} \sum \int \mathbf{q}_{j} n_{j}^{(1)} \frac{q d q}{4 \pi}
$$

Both methods require the inversion of the matrix $\Upsilon_{j j^{\prime}}^{(1)}$ to get the transport time (diffusion coefficient $(32))$

$$
\tau_{t r}=\sum_{j, j^{\prime}} q_{j} \Upsilon_{j j^{\prime}}^{(1)-1} q_{j^{\prime}} / \sum_{j} q_{j}^{2}
$$

The inversion of $\Upsilon_{j j^{\prime}}^{(1)}(58)$ cannot be performed unless the bulk scattering vertex $\widetilde{\Gamma}_{j j^{\prime}}\left(\mathbf{q}, \mathbf{q}^{\prime}\right)$ is known. Since we are not interested in the details of bulk scattering anyway, we should try to exclude this vertex from the equations for the effective transport time (63) by replacing it by observables - bulk relaxation and transport times.

Without the surface scattering term $W$ in Eq.(58), the effective scattering probability (frequency) is $m \widetilde{\Gamma} / 2$. The only reasonable way to proceed is to assume that $\widetilde{\Gamma}_{j j^{\prime}}\left(\mathbf{q}, \mathbf{q}^{\prime}\right)$ is a slowly varying function of momenta and discrete indices and, therefore, depends mostly on the angle between the vectors $\mathbf{q}$ and $\mathbf{q}^{\prime}$. This assumption is justified when the interaction radius $r_{0}$ is the smallest spatial scale in the problem, Eq.(7). Such vertex can be expanded in angular harmonics with constant coefficients,

$$
\tilde{\Gamma}_{j j^{\prime}}\left(\mathbf{q}, \mathbf{q}^{\prime}\right)=\frac{1}{2} \widetilde{\Gamma}^{(0)}+\widetilde{\Gamma}^{(1)} \cos \theta+\ldots
$$

where, since $\mathbf{q}$ is a $2 D$ vector, we use the Fourier, and not Legendre, expansion. The analogous $3 D$ expansion would include the expansion over the angle between $3 D$ vectors $(\pi j / L, \mathbf{q})$ and $\left(\pi j^{\prime} / L, \mathbf{q}^{\prime}\right)$ 


$$
\widetilde{\Gamma}_{j j^{\prime}}\left(\mathbf{q}, \mathbf{q}^{\prime}\right)=\widetilde{\Gamma}^{(0)}+\widetilde{\Gamma}^{(1)} \frac{\pi^{2} j j^{\prime} / L^{2}+\mathbf{q} \cdot \mathbf{q}^{\prime}}{\sqrt{(\pi j / L)^{2}+q^{2}} \sqrt{\left(\pi j^{\prime} / L\right)^{2}+q^{\prime 2}}}+\ldots
$$

The collision relaxation time, according to Appendix A (46), is expressed via the zeroth harmonic of the irreducible vertex as

$$
\frac{1}{\tau_{b}}=\frac{1}{2} S m \widetilde{\Gamma}^{(0)}
$$

Now Eq.(61) without $W$ can be solved analytically. The bulk transport time is expressed, as it should be, only via the zeroth and first harmonics of the vertex:

$$
\tau_{t r}^{b}=\tau_{b}\left[1+\frac{\widetilde{\Gamma}^{(1)}}{\widetilde{\Gamma}^{(0)}-\widetilde{\Gamma}^{(1)}} \frac{\left(\sum q_{j}\right)^{2}}{S \sum q_{j}^{2}}\right]
$$

This equation is a bit cumbersome because the quasi- $2 D$ quantized films differ from both truly $2 D$ or $3 D$ systems. In truly $2 D$ systems, when there is only one quantized level, $S=1$, the sums disappears and Eq.(67) reduces to the standard "transport" form

$$
\frac{1}{\tau_{t r}^{b}}=\frac{1}{2} m\left(\widetilde{\Gamma}^{(0)}-\widetilde{\Gamma}^{(1)}\right)
$$

In the opposite case of large number of levels, when the summation can be replaced by the integration, Eq. $(67)$ reduces to

$$
\frac{1}{\tau_{t r}^{b}}=\frac{m S}{2}\left(\widetilde{\Gamma}^{(0)}-\widetilde{\Gamma}^{(1)}\right)\left[1+\frac{\widetilde{\Gamma}^{(1)}}{\widetilde{\Gamma}^{(0)}}\left(\frac{3 \pi^{2}}{8}-1\right)\right]^{-1} .
$$

That this is still not a $3 D$ equation because we used Eq.(64) and not (65). In a truly quasiclassical $3 D$ case with the expansion over Legendre polynomials, the last factor in Eq.(69) becomes 1.

Using Eqs.(66),(67), one can replace the harmonics of the vertex $\widetilde{\Gamma}$ in the expressions for $\Upsilon_{j j^{\prime}}^{(1)}$ in Eq.(63) for the effective transport time with both bulk and boundary scattering via the observables $\tau_{b}$ and $\tau_{t r}^{b}$. Then the equation for the effective transport time $\tau_{t r}$ acquires the form (34). 


\section{FIGURE CAPTIONS FOR FIGURES}

Figure 1.Relative interference contribution $\chi_{j}$, Eq. (16), for $j=1 ; 5 ; 9$ as a function of the size of the surface inhomogeneities $p_{0} R$ for $p_{0} L=30$ and $p_{0} \mathcal{L}_{b}=100$

Figure 2. Relative interference contribution $\chi_{j}$, Eq. (16), for $j=1 ; 16 ; 31$ as a function of the bulk free path $p_{0} \mathcal{L}_{b}$ for $p_{0} L=100$ and $p_{0} R=50$

Figure 3. Relative interference contribution $\chi_{j}$, Eq. (16), for $j=1 ; 2 ; 3$ as a function of the film thickness $p_{0} L$ for $p_{0} R=5$ and $p_{0} \mathcal{L}_{b}=20$

Figure 4. Relative interference contribution to transport time, $\chi_{t r}$, Eq. (35), as a function of the size of the inhomogeneities $p_{0} R$ for $p_{0} \ell=0.1, p_{0} \mathcal{L}_{b}=10, \tau_{t r}^{b} / \tau_{b}=1.5$, and three values of the film thickness, $p_{0} L=5 ; 10 ; 20$

Figure 5. Relative interference contribution to transport time, $\chi_{t r}$, Eq. (35), as a function of the bulk free path $p_{0} \mathcal{L}_{b}$ at constant ratio $\tau_{t r}^{b} / \tau_{b}=1.5$. The inhomogeneities are characterized by $p_{0} R=10, p_{0} \ell=0.1$; the film thickness is $p_{0} L=5 ; 20$

Figure 6. Relative interference contribution to transport time, $\chi_{t r}$, Eq. (35), as a function of the film thickness $p_{0} L$ for $p_{0} \ell=0.1, p_{0} \mathcal{L}_{b}=20, \tau_{t r}^{b} / \tau_{b}=1.5$, and two values of the correlation radius, $p_{0} R=20 ; 50$

Figure 7. Dyson equation for $a$ ) impurity scattering; $b$ ) and $c$ ) impurity and surface scattering

Figure 8. Different types of averaged interaction diagrams. a) multiline connection; b) two intersecting second-order lines for impurity interaction; c) intersecting lines with impurity and wall scattering.

Figure 9. Main diagrams for the self-energy with the averaged wall scattering in the second order (two stars connected by the dashed line), the exact dressed impurity Green's functions (bold lines), and vertex $\Gamma$. 